APS/123-QED

\title{
Structural properties of a calcium aluminosilicate glass from molecular-dynamics simulations: A finite size effects study.
}

\author{
Patrick Ganster \\ Laboratoire des Verres UMR 5587, Université Montpellier II, \\ Place E. Bataillon, 34095 Montpellier, France \\ Laboratoire d'étude du Comportement à Long Terme, \\ CEA Valrhô, Marcoule, BP 17171, 30207 Bagnols sur Cèze cedex \\ Magali Benoit and Walter Kob \\ Laboratoire des Verres UMR 5587, Université Montpellier II, \\ Place E. Bataillon, 34095 Montpellier, France \\ Jean-Marc Delaye \\ Laboratoire d'étude du Comportement à Long Terme, \\ CEA Valrhô, Marcoule, BP 17171, 30207 Bagnols sur Cèze cedex
}

\begin{abstract}
We study a calcium aluminosilicate glass of composition $\left(\mathrm{SiO}_{2}\right)_{0.67^{-}}\left(\mathrm{Al}_{2} \mathrm{O}_{3}\right)_{0.12^{-}}(\mathrm{CaO})_{0.21}$ by means of molecular-dynamics (MD) simulations, using a potential made of two-body and threebody interactions. In order to prepare small samples that can subsequently be studied by firstprinciples, the finite size effects on the liquid dynamics and on the glass structural properties are investigated. We find that finite size effects affect the Si-O-Si and Si-O-Al angular distributions, the first peaks of the $\mathrm{Si}-\mathrm{O}, \mathrm{Al}-\mathrm{O}$ and $\mathrm{Ca}-\mathrm{O}$ pair correlation functions, the Ca coordination and the oxygen atoms environment in the smallest system (100 atoms). We give evidence that these finite size effects can be directly attributed to the use of three-body interactions.
\end{abstract}

PACS numbers: 75.40.Mg,61.43.Fs,31.15.Gg 


\section{INTRODUCTION}

Materials used in nuclear wastes confinement are made of glasses with complex compositions, and are based mainly on an alumino-boro-silicate network. These glasses have been studied for decades with the aim of testing their durability and their chemical resistance to water leaching, the process believed to be the limiting factor for their long time durability ${ }^{1}$ 2.3.4.5.6.7.8.9. When such glasses are altered by water leaching, the layers formed on the surface are hydrated silica-like porous gels enriched with aluminum and calcium. Although it is often argued that the protective property of this layer stops the alteration processes induced by the leaching, no real justification has yet been given 10,11,12,13.14 .

In calcium aluminosilicate glasses (CAS), silicon and aluminum are both network formers and have a tetrahedral coordination with oxygens $\frac{15}{}$. The tetrahedra are connected by shared oxygen atoms which are called bridging oxygens (BO) and they form an amorphous network in which $\mathrm{Ca}$ atoms are dispersed. Depending on the $\mathrm{Al}_{2} \mathrm{O}_{3} / \mathrm{CaO}$ ratio, $\mathrm{Ca}$ atoms play the role of modifiers if they create non-bridging oxygens (NBO) by breaking T-O-T linkages $(\mathrm{T}=\mathrm{Si} / \mathrm{Al})$ and/or they play the role of charge balancing, by neutralizing the $\mathrm{AlO}_{4}$ entities. In principle, the number of NBO atoms in a CAS glass is entirely determined by the $\mathrm{Al}_{2} \mathrm{O}_{3} / \mathrm{CaO}$ ratio and this number is directly related to the viscosity of the glass forming liquid. However it has been shown that this simple picture is not fully correct and that an excess number of $\mathrm{NBO}$ atoms exists even for an $\mathrm{Al}_{2} \mathrm{O}_{3} / \mathrm{CaO}$ ratio equal to one, which corresponds - in theory - to a perfect polymerized network. This excess number of NBO atoms could explain the viscosity anomaly measured in these systems $\frac{18}{}$ and could also be related to the existence of other structural units such as oxygen triclusters and/or highly coordinated $\mathrm{Si}$ and $\mathrm{Al}$ atoms. The calcium environment is also not very well defined in that it is quite difficult to attribute a specific coordination to this species. Nevertheless, in pure CAS systems, it was observed by X-ray and neutron spectroscopies that the distribution of oxygen neighbors around $\mathrm{Ca}$ atoms is independent of the composition ${ }^{16.17}$. Another feature observed in aluminosilicate glasses is the $\mathrm{Al} / \mathrm{Al}$ avoidance principle: As the formation of $\mathrm{Al}-\mathrm{O}-\mathrm{Al}$ linkages is energetically less favorable than $\mathrm{Si}-\mathrm{O}-\mathrm{Al}$ linkages, the occurrence of the former should be very low in CAS structures $19,20$.

Due to the experimental difficulties to study thin layers, an important first step to obtain a microscopic understanding of these materials can be achieved by the use of molecular- 
dynamics (MD) simulations. The aim of the present work is therefore to obtain a reasonable description of a CAS glass which composition is closed to the composition of the protective porous layer. However, in the case of such complex ternary systems, it is extremely difficult to find classical potentials that are able to give simultaneously a reliable description of the ionic and covalent bond lengths, of the bond angles and of the dynamics of the system. Alternatively, one can obtain a good description of such structural characteristics and of the dynamics of these complex systems from ab initio calculations. But in this case, the price to pay is the very limited size of the system (100 to 200 atoms) and the very short accessible length of the MD trajectories, thus making difficult the generation of disordered systems with good statistics on the one hand, and the equilibration of the samples at low temperatures on the other hand. In the aim of testing the classical potentials, we intend to use an approach in which the structure of a glass generated by classical MD is relaxed - and therefore tested - by ab initio MD. This approach has already been successfully used in the case of a $\mathrm{SiO}_{2}$ glass 21 and of a $\mathrm{Na}_{2} \mathrm{O}-4 \mathrm{SiO}_{2}$ glass ${ }^{22}$. Since ab initio simulations are limited to a rather small number of atoms, we first carried out a systematic study of the finite size effects on the aluminosilicate system of interest using a classical potential.

In this paper, we present the study of a calcium aluminosilicate glass by MD simulations in which we analyzed the dependence of the structural and dynamical properties on the system size. In the first section, technical details on the MD simulations are given. Subsequently the dynamics of the liquids are presented in section two and the third section is devoted to the results obtained on the glass structure. Finally we discuss and summarize the results.

\section{METHODOLOGY}

The chosen composition of the calcium aluminosilicate glass samples for this study is $67 \%$ $\mathrm{SiO}_{2}-12 \% \mathrm{Al}_{2} \mathrm{O}_{3}-21 \% \mathrm{CaO}$ in mole percent.

To describe the interatomic interactions, we used Born-Mayer-Huggins (BMH) and

Stillinger-Weber $(\mathrm{SW})^{23.24 .25}$ potentials with the modified parameters of Delaye ${ }^{26}$ which were obtained from an earlier study of complex multi-oxide glasses. The potentials include twobody and three-body terms. In Eq. (II) the two-body interaction is given, where $r_{i j}$ denotes the distance between atoms $i$ and $j$, and $A_{i j}, B_{i j}, C_{i j}$ and $D_{i j}$ are adjustable parameters. The first term of this pair potential corresponds to the Coulomb term, the second one to 
the core repulsion, and the third and fourth ones are referred to as multipolar terms:

$$
V_{i j}\left(r_{i j}\right)=\frac{q_{i} q_{j}}{r_{i j}}+A_{i j} \exp \left(-B_{i j} r_{i j}\right)-\left(\frac{C_{i j}}{r_{i j}}\right)^{6}+\left(\frac{D_{i j}}{r_{i j}}\right)^{8} .
$$

The Coulomb interactions were calculated using formal charges $q_{i}$ equal to +4 for $\mathrm{Si},-2$ for $\mathrm{O},+3$ for $\mathrm{Al}$ and +2 for $\mathrm{Ca}$, and the standard Ewald summation method with alpha $\mathrm{L}=7.2$ was used where $L$ is the size of the cubic box. Bond orientations in the CAS system can be described by adding a three-body term involving the O-Si-O, O-Al-O and Si-O-Si triplets. The three-body interaction is given by the product of a two-body interaction for each pair of the considered triplets by an angular strained force,

$$
V_{j i k}\left(r_{i j}, r_{i k}, \theta_{j i k}\right)=\lambda_{j i k} \exp \left(\frac{\gamma_{i j}}{r_{i j}-r_{i j}^{0}}+\frac{\gamma_{i k}}{r_{i k}-r_{i k}^{0}}\right) \times\left(\cos \left(\theta_{j i k}\right)-\cos \left(\theta_{j i k}^{0}\right)\right)^{2}
$$

where $r_{i j}$ and $r_{i k}$ denote the distances between atoms $i$ and $j$ (or atoms $i$ and $k$ ), $\theta_{j i k}$ is the angle between atoms $j, i$ and $k$, and $\lambda_{j i k}, \gamma_{i j}, \gamma_{i k}, r_{i j}^{0}$ and $r_{i k}^{0}$ are adjustable parameters. Tables \and I summarize the parameters of the two-body and the three-body interactions.

Newton's equations were integrated using a Velocity Verlet scheme with a time step of $1.8 \mathrm{fs}$ accepting a final deviation of $+7 \mathrm{~K}$ on the total energy for MD runs of 1 million steps.

Liquid samples of 100 and 200 atoms, generated using a random starting configuration, were equilibrated at different temperatures (from $\approx 3000 \mathrm{~K}$ to $\approx 7000 \mathrm{~K}$ ). The lowest temperature for which the silicon self-diffusion was at least $7 \AA$ after 1.8 ns of MD trajectory for both system sizes, was taken as the liquid equilibration temperature: it corresponds to roughly $4200 \mathrm{~K}$. Then, we generated and equilibrated independent liquid samples of different sizes at this temperature during 1.8 ns: 8 samples of 100 atoms, 4 samples of 200 atoms, 2 samples of 400 atoms, 2 samples of 800 atoms and 1 sample of 1600 atoms. The sizes of the corresponding boxes were respectively $11.32 \AA, 14.26 \AA, 17.97 \AA, 22.64 \AA$ and 28.52 $\AA$, corresponding to a density of $2.42 \mathrm{~g} . \mathrm{cm}^{-3}$, which is the equilibrium density obtained with this potential at this temperature. The experimental density for glasses of similar compositions at $300 \mathrm{~K}$ lies between 2.55 and $2.60 \mathrm{~g} / \mathrm{cm}^{327.28}$.

\section{DYNAMICS OF THE LIQUID}

The mean square displacements (MSD), $\left\langle r^{2}(t)\right\rangle=\left\langle\left(r_{i}(0)-r_{i}(t)\right)^{2}\right\rangle$, of each atomic species were calculated at different temperatures for the systems of 100 and 200 atoms. In order 
to obtain a good description of the dynamical properties, it appeared that an average over a larger number of samples was needed. Therefore these dynamical properties were studied only for the smallest system sizes of 100 and 200 atoms and using averages over 30 independent samples. Figure 1 represents the MSD of the various types of atoms in the 200-atoms liquid as a function of time in a logarithmic plot.

At short times, all curves exhibit a straight line with a slope of 2 which indicates that all atoms move ballistically. Then, after a transient regime, the atoms show a diffusive behavior which is characterized by straight lines with slopes of 1 . From the mean squared displacements at long times, the self-diffusion constants $D$ were extracted using Einstein's law:

$$
D=\lim _{t \rightarrow \infty} \frac{\left\langle r^{2}(t)\right\rangle}{6 t} .
$$

The resulting $D$ of the different species for systems of 200 atoms as a function of the inverse temperature are depicted in Fig. 2. The most diffusive element is $\mathrm{Ca}$, followed by $\mathrm{O}, \mathrm{Al}$ and $\mathrm{Si}$. It is interesting to note that the $\mathrm{Al}$ atoms follow the oxygen self-diffusion for all temperatures, despite the fact that, as $\mathrm{Si}$, it is a network former. This behavior resembles the one found in the $\mathrm{Al}_{2} \mathrm{O}_{3}-\mathrm{SiO}_{2}$ system ${ }^{29}$. Only at the lowest temperature the $\mathrm{Al}$ self-diffusion approaches the one of the silicon atoms. The activation energies of the self-diffusion of the $\mathrm{Si}, \mathrm{O}, \mathrm{Al}$ and $\mathrm{Ca}$ atoms were extracted from a linear fit of the curves in Fig. 2] (for 1/T greater than $2.310^{-4} \mathrm{~K}^{-1}$ ) and are equal to $6.4 \mathrm{eV}, 5.9 \mathrm{eV}, 6.5 \mathrm{eV}$ and $5.3 \mathrm{eV}$, respectively.

The evolution of the diffusion constants as a function of temperature as well as the values of the activation energies do not show any differences between the 100-atoms and 200-atoms liquids, suggesting that no size effects exist - at least up to 200 atoms - for the dynamical quantities. This is in apparent contrast with the results of previous studies carried out on supercooled liquids $s^{30.31}$ and in silica ${ }^{32}$ which shows strong finite size effects in the dynamics of the smallest samples. However, in order to study more deeply the finite size effects on the dynamics of the CAS liquids, more statistics would have been needed for systems of larger sizes as well. Since our main purpose is to study the size effects on the glass structural properties, we considered that this is beyond the scope of the present work. 


\section{STRUCTURAL ANALYSIS}

After cooling down all liquids from $4200 \mathrm{~K}$ to $300 \mathrm{~K}$ using a linear quench with a rate of $10^{13}{\mathrm{~K} . \mathrm{s}^{-1}}^{-1}$ and after a MD simulation of $150 \mathrm{ps}$ at $300 \mathrm{~K}$, we performed 1000 additional steps for the structural analysis.

\section{A. Radial distribution functions}

The radial distribution functions (RDF) were evaluated by means of Eq.(44) where $N_{\alpha}$ and $N_{\beta}$ denote the number of atoms for species $\alpha$ and $\beta$ and $V$ is the volume ${ }^{33}$ :

$$
g_{\alpha \beta}(r)=\frac{V}{4 \pi r^{2} N_{\alpha} N_{\beta}} \sum_{i \in\{\alpha\}} \sum_{j \in\{\beta\}}\left\langle\delta\left(r-r_{i j}\right)\right\rangle
$$

Figure 3 shows the radial distribution functions for Si-O, Al-O and $\mathrm{Ca}-\mathrm{O}$ pairs for all system sizes, with the 100-atoms system as dashed-lines. In all systems apart from the 100-atoms ones, the positions of the maxima of the Si-O and Al-O RDFs, which correspond to the most probable bond distances, are respectively equal to $1.60 \AA$ and $1.76 \AA$. These results

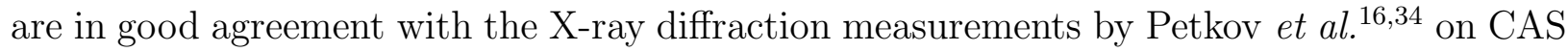
glasses of close compositions which give values of $1.63 \AA$ and $1.60 \AA$ for Si-O and $1.77 \AA$ and $1.75 \AA$ for Al-O. Because of the complexity of the Ca environment, more statistics would have been needed in order to refine the location of the maximum of the Ca-O RDF first peak. The latter can nevertheless be assigned approximately to $2.50 \AA$. The lower panels in Fig. 3 present zooms on the Si-O (a), Al-O (b), and $\mathrm{Ca}-\mathrm{O}$ (c) RDF first peaks. In these zooms, one can clearly notice that the systems of 100 atoms exhibit a different RDF than the ones of larger sizes since the $\mathrm{Si}-\mathrm{O}$ and $\mathrm{Al}-\mathrm{O}$ first peaks are narrower and higher. This indicates that the local order is more pronounced in the 100-atoms samples. The zoom on the Ca-O RDF clearly shows two peaks in the maximum. This double peak is not due to poor statistics but appears to be due to correlations that exist in all samples of 100 atoms.

In Fig. 4, we compare the Si-Ca, Al-Ca, Al-Al and Ca-Ca RDFs for the systems of 100(dashed lines) and 1600-atoms (bold lines). Due to the small number of $\mathrm{Al}$ and Ca atoms, the RDF curves are quite noisy for both system sizes. The determination of interatomic

distances is consequently quite difficult, particularly for the Ca-Ca distance. For systems of 
100-atoms, we can remark that the RDFs show several peaks that are absent in the larger system. Nevertheless, the RDFs of the 100-atoms system follow the global shape of the

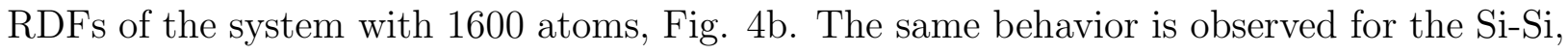
$\mathrm{Si}-\mathrm{Al}$ and $\mathrm{O}-\mathrm{O} \mathrm{RDFs}^{35}$.

The first peak of the Si-Ca and Al-Ca RDFs of the 1600-atoms system presents a double

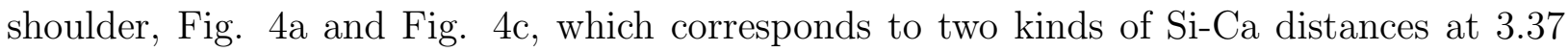
$\AA$ and $3.57 \AA$. A more pronounced double shoulder is also present on the first peak of the Al-Ca RDFs at $3.37 \AA$ and $3.57 \AA$. These double shoulders are due to the presence of nonbridging atoms (NBO) in the $\mathrm{SiO}_{4}$ or $\mathrm{AlO}_{4}$ groups. As mentioned in the introduction, the non-bridging oxygen (NBO) atoms are defined as oxygen atoms that have only one network former ( $\mathrm{Si}$ or $\mathrm{Al}$ ) as nearest neighbor. Here, these neighbors are counted using cutoff radii defined by the location of the minima after the first peaks in the Si-O and Al-O RDFs.

A detailed analysis of the Si-Ca RDF is presented in Fig. 5a, b and c. Figure 5a shows the first peak of the Si-O RDF for the different environments $\mathrm{Q}_{x}$ of the $\mathrm{Si}$ atoms. Since a $\mathrm{Q}_{x}$ atom is defined as a network former atom surrounded by $x$ bridging oxygen atoms, a $\mathrm{Q}_{x}$ tetra-coordinated network former is thus surrounded by (4-x) non-bridging oxygen atoms. Figure 5 clearly shows that the first peak of the Si-O RDF shifts to lower distances when the number of $\mathrm{NBO}$ is increased on the $\mathrm{SiO}_{4}$ groups (i.e. $x$ is decreased). The $\mathrm{Si}-\mathrm{O}$ bond length shifts from $1.60 \AA$ to $1.52 \AA$. Figure 5 b presents the Ca-O RDFs by differentiating BO and $\mathrm{NBO}$ atoms. A shorter Ca-O bond length is observed when the oxygen is non-bridging. By differentiating $\mathrm{Q}_{x} \mathrm{Si}$ atoms in the Si-Ca RDFs, Fig. 5 ; , it can be clearly concluded that the first shoulder, observed in the first peak of the Si-Ca RDF, is due to NBO atoms and the second to $\mathrm{BO}$ atoms, since the separation of the two peaks becomes more and more pronounced as the Si atoms are more and more surrounded by NBO atoms.

From the analysis of the 1600-atoms system, we summarize all interatomic distances of the CAS structure in Tab. III) the error bars being of the order of $5 \cdot 10^{-3} \AA$ in the case of $\mathrm{Si}-\mathrm{O}$ and $\mathrm{Al}-\mathrm{O}$ and between $10^{-2} \AA$ and $5 \cdot 10^{-2} \AA$ for the other distances. In the case of Si-Ca and Al-Ca, we took the positions of the first peak as a crude estimation of the distances, the RDF first peak being too noisy to allow a clear determination of the position of the maximum. Some of the values given in Tab. III can be compared with experimental distances obtained either on glasses or on crystalline analogous. Interatomic distances do not change dramatically with composition and one can compare directly the Si-O and the 
Al-O distances with the experimental values presented in Tab. [II]

In summary, we find clear finite size effects for the 100-atoms system which affect the first peaks of the $\mathrm{Si}-\mathrm{O}, \mathrm{Al}-\mathrm{O}$ and $\mathrm{Ca}-\mathrm{O}$ RDFs. These finite size effects suggest that the glass models become more locally organized for the smallest system size. For all the other system sizes, the RDFs give information on the local structure which is overall in agreement with experimental data except for the Ca-O bond length that is found to be slightly too large.

\section{B. Angular distributions and linkages}

In order to calculate angular distributions, we have defined cutoff radii using the location of the minima after the first peaks in the Si-O and Al-O RDFs, Fig. [3a. Figure 6 represents the Al-O-Al (a), Si-O-Si (b), Si-O-Al (c), O-Al-O (d) and O-Si-O (e) angular distributions for the systems of 100 and 1600 atoms. All other system sizes show similar distributions as the ones of the 1600-atoms system. Like the RDFs, the angular distributions of the systems of 100 atoms follow the general shape of the 1600-atoms ones but they present many small peaks that are due to the more ordered character of the systems. Particularly in the case of the Si-O-Si angular distribution, we have checked that these peaks are not due to statistics but correspond indeed to structural correlations that are present at the same position in all samples of 100 atoms.

In the 1600-atoms case, the O-Al-O and O-Si-O angular distributions, Fig. 66 $\mathrm{d}$ and e, show a quite symmetric shape centered at $107^{\circ}$ and $108^{\circ}$, respectively, which is in agreement with the values measured in amorphous silicates ${ }^{39}$. The $\mathrm{Si}-\mathrm{O}-\mathrm{Si}$ and $\mathrm{Si}-\mathrm{O}-\mathrm{Al}$ angular distributions, Fig. [6 and c, have maxima at $157^{\circ}$ and $148^{\circ}$ respectively, and exhibit an asymmetric shape with a tail toward small angles. The $\mathrm{Si}-\mathrm{O}-\mathrm{Si}$ angles are larger than the $\mathrm{Si}-\mathrm{O}-\mathrm{Al}$ ones which is consistent with the results of static ab initio calculations by Xiao and Lasaga 40 in which they showed that the equilibrium $\mathrm{Si}-\mathrm{O}-\mathrm{Si}$ angle of a $\mathrm{H}_{6} \mathrm{Si}_{2} \mathrm{O}$ cluster is larger than the $\mathrm{Si}-\mathrm{O}-\mathrm{Al}$ angle of a $\mathrm{H}_{6} \mathrm{AlSiO}$ cluster. The Al-O-Al angular distribution, Fig. $6 \mathrm{a}$, spreads from 90 to $180^{\circ}$ and does not present a distinctive shape with a clear maximum like in the $\mathrm{Si}-\mathrm{O}-\mathrm{Si}$ and $\mathrm{Si}-\mathrm{O}-\mathrm{Al}$ distributions. Because of the low number of Al-O-Al linkages in the system, a better statistics would be needed in order to refine the Al-O-Al distribution. That a few Al-O-Al linkages are present in the glass samples is consistent with the fact that the $\mathrm{Al} / \mathrm{Al}$ avoidance principle $\frac{19.20}{}$ is not necessarily fulfilled in amorphous aluminosilicates. 
In order to estimate to what extent the $\mathrm{Al} / \mathrm{Al}$ avoidance principle is violated in our glass samples, we computed the number of T-O-T' linkage concentrations ( $\mathrm{T}, \mathrm{T}$ ' $=\mathrm{Si}$ or $\mathrm{Al}$ ) that would be obtained if the $\mathrm{Si}$ and $\mathrm{Al}$ atoms were randomly distributed among all the possible network former sites in the 1600-atoms sample ${ }^{26}$. We then compared these concentrations with those found in the simulated glasses. If the $\mathrm{Si}^{+4}$ and $\mathrm{Al}^{+3}$ cations were randomly distributed among the network former sites of the CAS glass, the number of Al-O-Al linkages would be given by :

$$
N_{\mathrm{AlAl}}=N_{\mathrm{O}} \frac{N_{\mathrm{AlO}} \times\left(N_{\mathrm{AlO}}-1\right)}{\left(N_{\mathrm{SiO}}+N_{\mathrm{AlO}}\right) \times\left(N_{\mathrm{SiO}}+N_{\mathrm{AlO}}-1\right)}
$$

where $N_{\mathrm{AlAl}}$ is the number of Al-O-Al linkages, $N_{\mathrm{O}}$ is the number of bridging oxygens, $N_{\mathrm{SiO}}$ and $N_{\mathrm{AlO}}$ are the number of $\mathrm{Si}-\mathrm{O}$ and Al-O bonds, respectively. The probability to find an $\mathrm{Al}-\mathrm{O}-\mathrm{Al}$ linkage corresponds to the probability to pick out two $\mathrm{Al}-\mathrm{O}$ bonds among the different possibilities for the $\mathrm{Si}-\mathrm{O}$ and $\mathrm{Al}-\mathrm{O}$ bonds. In the system of 1600 atoms, the expectation value for the number of Al-O-Al linkages obtained from Eq. (55) is 62 whereas only $39 \mathrm{Al}-\mathrm{O}-\mathrm{Al}$ linkages are found in the simulated sample. This difference indicates that the $\mathrm{Al} / \mathrm{Al}$ avoidance exists for this kind of glass but is not completely fulfilled.

Fig. 7 shows the mean number of $\mathrm{Si}-\mathrm{O}-\mathrm{Si}, \mathrm{Si}-\mathrm{O}-\mathrm{Al}, \mathrm{Al}-\mathrm{O}-\mathrm{Al}$ linkages as well as the total number of linkages, divided by the total number of oxygen atoms, as a function of the system size. The variation of the number of linkages does not exceed $1 \%$ and remains inside the error bars, thus indicating that within the statistical errors no finite size effects can be detected on this quantity and that size effects only appear on the associated angular distributions.

\section{Coordination numbers}

In order to determine the $\mathrm{Si}, \mathrm{Al}$ and $\mathrm{Ca}$ coordination numbers, we used the same cutoff radii as the ones used for the angular distributions. We found that the local order of the $\mathrm{Si}$ and $\mathrm{Al}$ atoms is very well defined in that the atoms are always fourfold coordinated whatever the size of the system is. However, as the minimum of the Ca-O RDF is not defined very well, we calculated the Ca coordination distributions for ten different cutoff radii, chosen so that they sample regularly the region of the minimum (see inset of Fig. [). Only five of these

distributions, as well as their average, are shown in Fig. 8 for the 1600-atoms system. The 
coordination distribution of the Ca atoms is strongly dependent on the choice of the cutoff radii and therefore caution should be used while discussing the average Ca coordination number.

The averaged Ca coordination distributions for the different system sizes are presented in Fig. 9. From this figure, we can see that the Ca atoms have multiple local environments, the number of oxygen surrounding the Ca atoms ranging from 4 to 12 . The maxima of these distributions are located between 7 and 8 except for the 100-atoms systems. In that case, the distribution seems to be shifted toward smaller numbers, to be more asymmetric and to have a maximum located at 6 . From experimental measurements, the coordination of the Ca atoms were estimated to be equal to 7 in $\mathrm{WAXS}^{17}$ and to 5.2 in X-ray diffraction ${ }^{34}$, and to 6 by Car-Parrinello simulations in a CAS liquid of 100 atoms $\stackrel{41}{ }$. Therefore it seems that our models slightly overestimate the Ca mean coordination and that this overestimation is compensated by finite size effects in the 100-atoms case.

\section{Bridging oxygens, non-bridging oxygens, and oxygen triclusters}

As defined in Sec. IVA the non-bridging oxygen (NBO) atoms are oxygen atoms that have only one network former ( $\mathrm{Si}$ or $\mathrm{Al}$ ) as nearest neighbor. These neighbors are counted using the cutoff radii used for the angular distributions as well as for the coordination numbers.

The expected number of NBO atoms in the system can be estimated from simple stoichiometry arguments, if the system is supposed to be made of perfect tetrahedra with only 2-fold oxygen atoms. In that case, the number of NBO atoms is simply given by:

$$
N_{\mathrm{NBO}}=2 \times N_{\mathrm{Ca}}-N_{\mathrm{Al}}
$$

Following this argument, the percentage of NBO atoms in all systems should be equal to $9.52 \%$, if the $\mathrm{Ca}^{2+}$ ions fully play their charge balancing role. However, in the simulated samples, we always find an excess number of NBO atoms. Fig. 10a shows the percentage of NBO as a function of the system size and this percentage is clearly located between 10.7 $\%$ and $11.8 \%$. The excess number of NBO atoms could be due to the use of a too high quench rate, however recent ${ }^{17} \mathrm{O}$ NMR experiments on calcium and sodium aluminosilicate glasses $\frac{18,45}{2}$ revealed the presence of excess NBO atoms as well. 
One can observe a slight size dependence for this number indicating that, for larger systems, a larger number of NBO atoms is found. It is furthermore interesting to note that a large majority of NBO atoms are located on the Si tetrahedra and only a few percent of them on $\mathrm{Al}$ tetrahedra (between 1.7 and $8.0 \%$ of the $\mathrm{NBO}$ atoms are located on $\mathrm{Al}$ tetrahedra). This result is in agreement with X-ray diffraction experiments by Petkov et $a l{ }^{34}$ who observed that the NBO atoms are exclusively located on Si tetrahedra in CAS glasses. One can also estimate the number of NBO atoms that are located on a given $\mathrm{Si}$ tetrahedron, i.e. if the $\mathrm{Si}$ tetrahedron is a $\mathrm{Q}_{4}$ (no NBO), a $Q_{3}$ (one $\mathrm{NBO}$ ), a $\mathrm{Q}_{2}$ (two NBO) etc. This information is given in Tab. IV] As it can be seen, there are no clear size effects on the relative number of $\mathrm{Q}_{x}$ and most of the Si tetrahedra with NBO atoms are in the $\mathrm{Q}_{3}$ conformation.

It has been proposed that the excess number of oxygen atoms could be compensated by oxygen triclusters, i.e. oxygen atoms which are bonded to three network former atoms $\frac{18,41}{}$. We therefore evaluated the number of oxygen triclusters in our samples and we found that the percentage varies between $1 \%$ and $1.5 \%$, Fig. 10k. Again, this percentage shows an increase with the system size as for the number of NBO atoms, Fig. 10a, even if the error bars are quite large for the small system sizes. Finally the number of BO atoms (which are not triclusters) was also evaluated and the resulting percentage is depicted in Fig. [10b.

In order to determine if there exists any particular trend in the formation of tricluster types $\left(\mathrm{OSi}_{3}, \mathrm{OSi}_{2} \mathrm{Al}, \mathrm{OSiAl}_{2}\right.$ and $\left.\mathrm{OAl}_{3}\right)$, we evaluated the percentage of these units in the case of a random distribution of the $\mathrm{Si}$ and $\mathrm{Al}$ atoms among the possible network former sites. Let $N_{\mathrm{O} 3}$ be the number of oxygens triclusters. Then there are $3 \times N_{\mathrm{O} 3}$ bonds between the oxygen triclusters and the three network former neighbors. Among these bonds, we can distinguish the number of $\mathrm{Si}-\mathrm{O} 3$ bonds, given by $N_{\mathrm{Si}-\mathrm{O} 3}$, and the number of Al-O3 bonds, given by $N_{\mathrm{Al}-\mathrm{O} 3}$. We consider the entity formed by the oxygen tricluster and its three neighbors as an ensemble of three $\mathrm{T}-\mathrm{O}$ bonds $(\mathrm{T}=\mathrm{Si}$ or $\mathrm{Al})$. Then, the probability to pick out two Si-O3 bonds and one Al-O3 bond among the complete ensemble of $3 \times N_{\mathrm{O} 3}$ bonds can be written for the $\mathrm{OSi}_{2} \mathrm{Al}$ triclusters, using the standard combinatory notations:

$$
\frac{C_{N_{\mathrm{Si}-\mathrm{O} 3}}^{2} \times C_{N_{\mathrm{Al}-\mathrm{O} 3}}^{1}}{C_{3 \times N_{\mathrm{O} 3}}^{3}}
$$

where

$$
C_{k}^{n}=C(n, k)=\frac{n !}{k !(n-k) !}
$$


The derivation of this formula for the other tricluster types is trivial. When we compare the expected values for a random distribution of $\mathrm{Si}$ and $\mathrm{Al}$ among the possible sites to the values obtained in the simulated sample, an excess of $\mathrm{OSiAl}_{2}$ is observed in the 1600atoms system since $13 \mathrm{OSiAl}_{2}$ triclusters are found instead of 8.2, the randomly expected. This excess number of $\mathrm{OSiAl}_{2}$ is accompanied by a deficit of $\mathrm{OSi}_{2} \mathrm{Al}$ and $\mathrm{OAl}_{3}$ triclusters, compared to a random distribution of $\mathrm{Si}$ and $\mathrm{Al}$. This result suggests a possible charge compensation role of the oxygen triclusters and could partly explain the violation of the $\mathrm{Al} / \mathrm{Al}$ avoidance rule since $\mathrm{Al}-\mathrm{O}-\mathrm{Al}$ linkages are present in the $\mathrm{OSiAl}_{2}$ triclusters.

\section{E. Rings distribution}

In silicate systems, rings are defined as loops of $\mathrm{T}-\mathrm{O}$ links $(\mathrm{T}=\mathrm{Si}$ or $\mathrm{Al})$ and the ring size distribution is a measure of the intermediate range order. In the case of aluminosilicate glasses, we defined a ring as the smallest loop composed of T-O links. Fig. 11 presents the

ring size distributions computed for all system sizes. The distributions extend from rings of size 3 to rings of size 9 and they present a maximum located at 6 for all system sizes. As the size of the system decreases, the size of the box becomes comparable to the size of the rings and only few large rings can be found. However it is interesting to note that the distribution maxima do not shift to smaller values for the small systems, which indicates that the finite size effects are not significant on this quantity. This result is interesting since we know from the $\mathrm{RDFs}^{35}$ that correlations exist in the 1600 -atoms system up to $8 \AA$ at least, which is much larger than half the simulation box length of the 100-atoms system. Yet the fact that the medium range correlations are not taken into account in the 100-atoms system does not seem to affect the ring size distribution.

As the maximum of the rings distribution is 6 for pure silica ${ }^{42}$ and 5 for aluminosilicate $(\mathrm{AS} 2)^{29}$, we do not denote an effect of the presence of aluminum or calcium atoms in the rings distribution which is silica-like.

\section{F. Static structure factor}

In order to compare the global structure of the simulated systems with the one of real systems, we computed the neutron static structure factors. This was done using the relations: 


$$
\begin{gathered}
S_{n}(q)=\frac{1}{\sum_{\alpha} N_{\alpha} b_{\alpha}^{2}} \sum_{\alpha \beta} b_{\alpha} b_{\beta} S_{\alpha \beta}(q), \\
\text { with } S_{\alpha \beta}(q)=\frac{f_{\alpha \beta}}{N} \sum_{l=1}^{N_{\alpha}} \sum_{m=1}^{N_{\beta}}\left\langle\exp \left(i \mathbf{q} \cdot\left(\mathbf{r}_{l}-\mathbf{r}_{m}\right)\right)\right\rangle
\end{gathered}
$$

where $\mathbf{q}$ is the scattering vector, $N$ the number of atoms, $N_{\alpha}$ and $N_{\beta}$ the number of atoms of species $\alpha$ and $\beta$, respectively. The factor $f_{\alpha \beta}$ is equal to 0.5 for $\alpha \neq \beta$ and equal to 1.0 for $\alpha=\beta, b_{\alpha}$ and $b_{\beta}$ are the neutron scattering lengths of species $\alpha$ and $\beta$, and are equal to $4.149,5.803,3.449,4.700 \mathrm{fm}$ for $\mathrm{Si}, \mathrm{O}, \mathrm{Al}$ and $\mathrm{Ca}$, respectively ${ }^{43}$.

Figure 12 shows the comparison of the neutron structure factors calculated for the systems of 100 and 1600 atoms. Even if the 100-atoms $S_{n}(\mathrm{q})$ is noisier, there are no perceptible differences between the 100- and the 1600-atoms structure factors. From the partial structure factors, the principal contributions to the $S_{n}(\mathrm{q})$ first peak appear to be due to the $\mathrm{Si}-\mathrm{Si}$, Si-O, Al-O and O-O correlations (see Fig. 13). Concerning the second peak, the main contributions can be attributed to $\mathrm{Si}-\mathrm{Si}, \mathrm{Si}-\mathrm{Al}, \mathrm{O}-\mathrm{O}$ and $\mathrm{Ca}-\mathrm{O}$ correlations. It is, however, more difficult to make a clear relation between the other peaks and the partial correlations.

On the other hand, in the 1600-atoms $S_{n}(q)$, one can notice the existence of a very small pre-peak around $0.7 \AA^{-1}$ (denoted by a vertical dashed line in Fig. 13) which appears to be due to Si-Si, Si-O and O-O correlations. Since the Al-Al correlations do not contribute to this pre-peak, it is very likely that this feature is indeed due to the presence of the Ca atoms which induce the existence of pockets or channels and consequently, the oorganisation of the matrix on large scales. This type of long-range organisation has already been observed in sodo-silicate glasses and melts $\stackrel{44}{ }$.

Since no experimental data were available for the specific composition of the present work, in order to compare the computed $S_{n}$ (q) with experimental data, we had to generate a glass with a slightly different composition (60\% $\left.\mathrm{SiO}_{2}, 10 \% \mathrm{Al}_{2} \mathrm{O}_{3}, 30 \% \mathrm{CaO}\right)$. This new simulation was performed on a system of 800 atoms following the same procedure as described beforehand. The comparison between the computed and experimental structure factors from Ref 17 is presented in Fig. 14. The agreement is good both for the positions of the peaks and for their intensities except at large q where irregularities do not permit to superpose the shoulder after the fourth peak.

The difference between the two computed compositions is the $\mathrm{SiO}_{2} / \mathrm{CaO}$ ratio, which is 
lower in the case of the experimental comparison. The differences between the structure factors at the two compositions, Fig. 12 and Fig. 14, mainly concern the two first peaks. In the experimental composition the first peak is lower and the second one higher than in the initial studied composition. This shift is due to the larger quantities of $\mathrm{Ca}$ in the experimental composition and has already been observed in a neutron scattering study of calcium aluminosilicate glasses of different compositions ${ }^{17}$.

\section{DISCUSSION}

The set of interatomic potentials used to simulate the calcium aluminosilicate glasses leads to structures that reproduce a series of experimental features if the studied sample contains at least 200 atoms.

Firstly, the simulated neutron structure factor is globally in agreement with the experimental one although some of the first neighbor distances are not correctly reproduced. Recent reverse Monte Carlo calculations ${ }^{26}$ carried out on glasses of several compositions have suggested that the distance discrepancies in the local ionic environments could explain the slight differences between the simulated and experimental structure factors. By stretching the local Si-O distances by $0.02 \AA$ and reducing the Al-O and Ca-O distances by respectively $0.05 \AA$ and $0.1 \AA$, these differences could be removed.

Secondly, the $\mathrm{Al} / \mathrm{Al}$ avoidance rule is qualitatively reproduced but we have no quantitative experimental results to compare with simulations. The fact that the avoidance rule is not completely obeyed has already been suggested in recent NMR experiments ${ }^{45}$. The authors predict that there still exist $\mathrm{Al}-\mathrm{O}-\mathrm{Al}$ linkages in a sodium aluminosilicate glass with $\mathrm{Si} / \mathrm{Al}=$ 0.7 and in a calcium aluminosilicate glass with $\mathrm{Si} / \mathrm{Al}=0.5$ which suggests that the respective concentrations in $\mathrm{Si}-\mathrm{O}-\mathrm{Si}, \mathrm{Si}-\mathrm{O}-\mathrm{Al}$ and $\mathrm{Al}-\mathrm{O}-\mathrm{Al}$ are dependent on the energetic cost of the local configurations surrounding each linkage.

Finally, another interesting structural feature concerns the existence of oxygen triclusters. If we consider that each Ca atom is "used" to compensate the charged $\mathrm{AlO}_{4}$ tetrahedra and that the remaining $\mathrm{Ca}$ ions create non-bridging oxygens, the predicted concentration of nonbridging oxygens is lower than the simulated ones. This result means that the $\mathrm{Ca}$ atoms are not completely consumed by the $\mathrm{AlO}_{4}$ tetrahedra, and part of the $\mathrm{AlO}_{4}$ tetrahedra are charge-balanced by the oxygen triclusters. The existence of the latter, proposed originally 
in Ref. $\stackrel{46}{ }$, has not been directly observed but is deduced from ${ }^{17} \mathrm{O}$ NMR measurements in aluminosilicate glasses. The existence of $5 \%$ of non-bridging oxygen in a $\mathrm{CaAl}_{2} \mathrm{Si}_{2} \mathrm{O}_{8}$ glass, detected by ${ }^{17} \mathrm{O}$ NMR spectroscopy, suggests also the presence of oxygen triclusters ${ }^{47}$. Previous simulations based on similar potentials have also predicted the existence of oxygen triclusters in a $\mathrm{CaAl}_{2} \mathrm{Si}_{2} \mathrm{O}_{8}$ glass ${ }^{48}$.

However for the glass models containing 100 atoms, the structural characteristics are quite different indicating that these systems are subject to finite size effects. To test whether these size effects are due to the used potential or to the complexity of the glass, we performed simulations on a 78-atoms system of pure a- $\mathrm{SiO}_{2}$ by using two different potentials: the present one detailed in section II and the so-called BKS potential derived by van Beest $a l . \stackrel{49}{ }$. Whereas the $\mathrm{SiO}_{2}$ glass obtained with the BKS potential did not show any finite size effects, the size effects observed in the present work on the RDF first peaks and on the angular distributions were still present with the potential described in section III The main differences between the two potentials reside in the fact that the BKS potential comprises partial charges for the $\mathrm{Si}$ and $\mathrm{O}$ ions and does not include three body terms.

In order to determine whether the size effects in the CAS system are induced by the presence of the three body terms, we have performed calculations without the three body terms. By neglecting the angular terms, we do not obtain a correct structure, since a large number of miscoordinated silicon atoms are found. However the structural quantities concerned by the finite size effects can still be examined. For instance, the first peaks of the Si-O and Al-O radial distribution functions for systems of 100 and 1600 atoms have been compared without the three body terms and we clearly observe the disappearance of the size effects on those RDFs. The finite size effects also disappear in the angular distributions. These considerations allow us to clarify the effect of the angular terms on the calculated glassy structures. The enhancement of the local order observed for the small systems is due to the three body terms: it completely disappears when these terms are removed.

However, for the smallest systems, finite size effects are still present since the structure on distances beyond $L / 2$ is influenced by the periodic boundary conditions. Therefore it is not possible to investigate the intermediate range order, which in these type of materials extends

up to 10-15 $\AA$, in systems that are smaller than $10 \AA$. These effects are not responsible of the enhancement of the local structure, but rather of the modification of the structure factor at low $q$. These effects are somehow trivial and can be easily be taken into account. 


\section{CONCLUSION}

In this work, we have presented a systematic study of the liquid dynamics and the structural properties of a calcium aluminosilicate glass as a function of the system size. This study was carried out by means of molecular dynamics simulations using a potential made of two-body and three-body interactions. The results show that finite size effects exist for the smallest system size (100 atoms) and affect a large part of structural characteristics: the Si-O-Si and Si-O-Al angular distributions, the Ca coordination numbers, the first peaks of the Si-O, Al-O and $\mathrm{Ca}-\mathrm{O}$ pair distribution functions, and the relative number of bridging oxygens, non-bridging oxygens and oxygens triclusters.

Most of these size effects were clearly attributed to the presence of three-body interactions in the potential. Interestingly, no finite size effects were visible in the relative percentages of $\mathrm{Al}-\mathrm{O}-\mathrm{Al}$ linkages and on the ring size distribution. For the larger system size, the structural characteristics were compared to experimental data when possible and a correct agreement was obtained for the Si-O and Al-O interatomic distances and for the neutron static structure factors. However, the Si-O-Si and Si-O-Al mean angles and the Ca coordination numbers were found to be slightly too large.

In conclusion, the present work gives confidence in the fact that the local structure of relatively complex glasses can be studied by the means of ab initio simulations carried out on relatively small samples (200 atoms). On the other hand, it also gives indications on the weaknesses of the presently used classical potential and guidelines for possible improvements.

1 P. Aagaard and H.C. Helgeson, Am. J. Science 282, 237 (1982).

2 E.H. Oelkers, Geochim. and Cosmochim. Acta 65, 3703 (2001).

3 B. Grambow, Mat. Res. Soc. Symp. Proc. 44, 15 (1984).

4 Z. Boksay, G. Bouquet, and S. Dobos, Physics and Chemistry of Glasses 8, 140 (1967).

5 Z. Boksay, G. Bouquet, and S. Dobos, Physics and Chemistry of Glasses 9, 69 (1968).

6 S.C. Kohn, Mineral. Mag. 64, 389 (2000).

7 F. Pacaud, N. Jacquet-Francillon, A. Terki and C. Fille, Mat. Res. Soc. Symp. Proc. 127, 105 (1988).

8 E.Y. Vernaz and J.L. Dussossoy, Appl. Geochem. Suppl. 1, 13 (1992). 
9 E.Y. Vernaz and N. Godon, Mat. Res. Soc. Symp. Proc. 257, 37 (1992).

10 S.-B. Xing, A.C. Buechele, and I.L. Pegg, Mat. Res. Soc. Symp. Proc. 333, 541 (1994).

11 J.C. Dran, J.C. Petit, and C. Brousse, Nature 319, 485 (1986).

12 S. Gin, Mat. Res. Soc. Symp. Proc. 663, 207 (2000).

13 F. Angeli, D. Boscarino, S. Gin, G. Della Mea, B. Boizot, and J.-C. Petit, Physics and Chemistry of Glasses 42, 279 (2001).

14 J.A. Tossel and G. Saghi-Szabo, Geochim. Cosmochim. Acta 61, 1171 (1997).

15 Z. Wu et al., Phys. Rev. B 60, 9216 (1999).

16 V. Petkov, S.J.L. Billinge, S.D. Shastri, B. Himmel, Phys. Rev. Lett. 85, 3436 (2000).

17 L. Cormier, D.R. Neuville, and G. Calas, J. Non-Cryst. Solids 274, 110 (2000).

18 J.F. Stebbins and Z. Xu, Nature 390, 60 (1997).

19 E.R. Meyers, V. Heine, and M.T. Dove, Physics and Chemistry of Minerals 25, 457 (1998).

20 J.F. Stebbins, Nature 330, 13 (1987).

21 M. Benoit, S. Ispas, P. Jund, and R. Jullien, Euro. Phys. J. B 13, 631 (2000).

22 S. Ispas, M. Benoit, P. Jund and R. Jullien, Phys. Rev. B 64, 214206 (2001).

23 P. Cheeseman, L.V. Woodcock, and C.A. Angell, J. Chem. Phys. 65, 1565 (1976).

24 T.F. Soules, J. of Non-Cryst. Solids 49, 29 (1982).

25 F.H. Stillinger and T.A. Weber, Phys. Rev. A 31, 4234 (1985).

26 J.M. Delaye, L. Cormier, D. Ghaleb, G. Calas, J. Non-Cryst. Solids 290, 293 (2001).

27 N.P. Bansal and R.H. Doremus, Handbook of Glass Properties, Academic Press. Inc.

28 C. Huang and E.C. Behrman, J. Non-Cryst. Solids 128, 310 (1991).

29 A. Winkler, J. Horbach, W. Kob, K. Binder, J. Chem. Phys. 120, 384 (2004).

30 B. Doliwa and A. Heuer, J. of Phys.: Cond. Mat. 15, S849 (2003).

31 K. Kim and R. Yamamoto, Phys. Rev. E 61, R41 (2000).

32 J. Horbach, W. Kob, K. Binder, and C.A. Angell, Phys. Rev. E 54, R5897 (1996).

33 J.-P. Hansen and I.R. McDonald, Theory of Simple Liquids (Academic, London, 1986).

34 V. Petkov, Th. Gerber, and B. Himmel, Phys. Rev. B 58, 11982 (1998).

35 P. Ganster, Ph. D. thesis, Université Montpellier II (2004).

36 B. Himmel, J. Weigelt, Th. Gerber, and M. Nofz, J. Non-Cryst. Solids 136, 27 (1991).

37 P. McMillan, B. Piriou, and A. Navrotsky, Geochim. Cosmochim. acta 46, 2021 (1982).

38 G. Calas, G.E. Brown, G.A. Waychunas, and J. Petiau, Phys. and Chem. of Minerals 15, 19 
(1987).

39 R.F. Pettifer, R. Dupree, I. Farman, and U. Sternberg, J. Non-Cryst. Solids 106, 408 (1988).

40 Y. Xiao and A.L. Lasaga, Geochim. Cosmochim. Acta 58(24), 5379 (1994).

41 M. Benoit, S. Ispas, and M. Tuckerman, Phys. Rev. B 64, 224205 (2001).

42 K. Vollmayr, W. Kob, and K. Binder, Phys. Rev. B 54, 15808 (1996).

43 www.ncnr.nist.gov/resources/n-lenths/elements/

44 J. Horbach, W. Kob, and K. Binder, J. Chem. Geol. 174, 87 (2001).

45 J.F. Stebbins, S.K. Lee, and J.V. Oglesby, Am. Mineral. 84, 983 (1999).

46 E.D. Lacy, Physics and Chemistry of Glasses 4, 234 (1963).

47 J.F. Stebbins and Z. Xu, Science 390, 60 (1997).

48 D. Nevins and F.J. Spera, Am. Mineral. 83, 1220 (1998).

49 B.W.H. van Beest, G.J. Kramer, and R.A. van Santen, Phys. Rev. Lett. 64, 1955 (1990). 
TABLE I: Parameters of the two-body potentials.

\begin{tabular}{|c|c|c|c|c|}
\hline atomic pair & $A_{i j}[\mathrm{eV}]$ & $B_{i j}[\AA]$ & $C_{i j}[\mathrm{eV}]$ & $D_{i j}[\mathrm{eV}]$ \\
\hline $\mathrm{Si}-\mathrm{Si}$ & 875.9321 & 3.448 & & \\
\hline $\mathrm{Si}-\mathrm{O}$ & 1044.9519 & 3.049 & & \\
\hline $\mathrm{Si}-\mathrm{Al}$ & 956.3850 & 3.448 & & \\
\hline $\mathrm{Si}-\mathrm{Ca}$ & 4000.4894 & 3.448 & & \\
\hline $\mathrm{O}-\mathrm{O}$ & 369.2783 & 2.857 & & \\
\hline $\mathrm{O}-\mathrm{Al}$ & 1725.0881 & 3.448 & & \\
\hline $\mathrm{O}-\mathrm{Ca}$ & 8973.4045 & 3.448 & 539.9580 & 884.2333 \\
\hline $\mathrm{Al}-\mathrm{Al}$ & 1039.5842 & 3.448 & & \\
\hline $\mathrm{Al}-\mathrm{Ca}$ & 4326.2957 & 3.448 & & \\
\hline $\mathrm{Ca}-\mathrm{Ca}$ & 17897.5012 & 3.448 & & \\
\hline
\end{tabular}

TABLE II: Parameters of the three-body potentials.

\begin{tabular}{ccccccc}
\hline \hline atomic triplet & $\lambda_{j i k}[\mathrm{eV}]$ & $\gamma_{i j}[\AA]$ & $\gamma_{i k}[\AA]$ & $r_{i j}^{0}[\AA]$ & $r_{i j}^{0}[\AA]$ & $\theta_{j i k}[$ degree $]$ \\
\hline O-Si-O & 149.7960 & 2.6 & 2.6 & 3.0 & 3.0 & 109.5 \\
O-Al-O & 149.7960 & 2.6 & 2.6 & 3.0 & 3.0 & 109.5 \\
Si-O-Si & 6.2415 & 2.0 & 2.0 & 2.6 & 2.6 & 160.0 \\
\hline \hline
\end{tabular}


TABLE III: Interatomic distances in the CAS glass for a system of 1600 atoms at $300 \mathrm{~K}$, as extracted from the positions of the first peak maxima in the corresponding $g_{\alpha \beta}(r)$.

\begin{tabular}{ccc}
\hline \hline & & Interatomic distance $[\AA]$ \\
atomic pair & This work & Experimental \\
\hline Si-Si & 3.18 & $3.09^{a}$ \\
Si-O & 1.60 & $1.63^{b}, 1.60^{c}$ \\
Si-Al & 3.24 & \\
Si-Ca & 3.50 & $2.65^{a}$ \\
O-O & 2.59 & $1.77^{b}, 1.75^{c, d}, 1.74^{e}$ \\
O-Al & 1.76 & $2.32^{c}, 2.40^{f}$ \\
O-Ca & 2.50 & \\
Al-Al & 3.25 & \\
Al-Ca & 3.50 & \\
Ca-Ca & - & \\
\hline \hline
\end{tabular}

${ }^{a}$ Reference ${ }^{36}$

${ }^{b}$ Reference ${ }^{34}$

${ }^{c}$ Reference ${ }^{16}$

${ }^{d}$ References ${ }^{37}$

${ }^{e}$ Reference ${ }^{38}$

${ }^{f}$ Reference ${ }^{17}$

TABLE IV: Percentages of the Si tetrahedra in the $\mathrm{Q}_{x}$ environment for all system sizes.

\begin{tabular}{lrrrrr}
\hline \hline & & & & \\
& & & System size & \\
& 100 & 200 & 400 & 800 & 1600 \\
\hline $\mathrm{Q}_{1}$ & 0 & 0.6 & 0 & 0.6 & 0 \\
$\mathrm{Q}_{2}$ & 2.9 & 1.7 & 2.3 & 3.4 & 3.7 \\
$\mathrm{Q}_{3}$ & 22.7 & 23.8 & 26.1 & 23.9 & 25.3 \\
$\mathrm{Q}_{4}$ & 74.4 & 73.9 & 71.6 & 72.1 & 71.0 \\
\hline \hline
\end{tabular}




\section{FIGURE CAPTIONS}

FIgURE 1 : Time and temperature dependence of the mean squared displacements of $\mathrm{Si}$, $\mathrm{O}, \mathrm{Al}$ and $\mathrm{Ca}$ atoms for the 200-atoms systems at $3300 \mathrm{~K}$ and $5800 \mathrm{~K}$.

FiguRE 2 : Inverse temperature dependence of the self diffusion constants for $\mathrm{Si}, \mathrm{O}, \mathrm{Al}$, and $\mathrm{Ca}$ atoms in the 200-atoms liquids.

Figure 3 : (a) Si-O, Al-O and $\mathrm{Ca}-\mathrm{O}$ radial distribution functions for systems of 100 (dashed lines), 200, 400, 800 and 1600 atoms (bold lines). (b) Zoom on the first peak of the Si-O radial distribution function. (c) Zoom on the first peak of the Al-O radial distribution function. (d) Zoom on the first peak of the Ca-O radial distribution function.

Figure 4 : (a) Si-Ca, (b) Ca-Ca, (c) Al-Ca and (d) Al-Al radial distribution functions for the systems of 100 (dashed lines) and 1600 atoms (bold lines).

Figure 5 : (a) Si-O radial distribution functions for different Si environments $Q_{2}, Q_{3}, Q_{4}$ and for all $\mathrm{Si}$ atoms. (b) Ca-O radial distribution functions for different oxygen atom types BO, NBO and for all oxygen atoms. (c) Si-Ca radial distribution functions for different Si environments $Q_{2}, Q_{3}, Q_{4}$ and for all Si atoms. All RDFs are computed for the 1600-atoms system.

Figure 6 : Angular distributions of the (a) Al-O-Al, (b) Si-O-Si, (c) Si-O-Al, (d) O-Al-O and (e) O-Si-O angles for systems of 100 (dashed lines) and 1600 (bold lines) atoms.

FiguRE 7 : Number of linkages divided by the number of oxygen atoms as the function of the system size: (a) Si-O-Si, (b) Si-O-Al, (c) Al-O-Al and (d) sum of Si-O-Si, Si-O-Al and $\mathrm{Al}-\mathrm{O}-\mathrm{Al}$.

Figure 8 : Averaged probability distribution for finding $\mathrm{N}$ oxygen atoms around the calcium atoms in a system of 1600 atoms and for different cutoff radii. The inset shows schematically how the cutoff radii were chosen around the minimum after the first peak of the $\mathrm{Ca}-\mathrm{O}$ radial distribution function. 
Figure 9 : Probability distribution for finding $\mathrm{N}$ oxygen atoms around the calcium atoms for systems from 100 to 1600 atoms.

Figure 10 : Concentration of (a) NBO atoms, (b) BO atoms and (c) oxygen triclusters as the function of the system size.

Figure 11 : Probability of finding rings of N tetrahedra for systems of 100, 200, 400, 800 and 1600 atoms.

Figure 12 : Static neutron structure factors for 100-atoms (bold line) and 1600-atoms (circles) systems. The scattering lengths were taken to be equal to $b_{S i, O, A l, C a}=4.149,5.803$, $3.449,4.700 \mathrm{fm}$.

FiguRE 13 : Partial structure factors for the 1600-atoms system. The curves have been shifted upward and downward for clarity and the vertical dashed line denotes the position of the pre-peak.

Figure 14 : Comparison of the experimental (from Ref: $\frac{17}{}$ ) and calculated neutron structure factors for a 800-atoms CAS glass of composition $60 \% \mathrm{SiO}_{2}-10 \% \mathrm{Al}_{2} \mathrm{O}_{3}-30 \%$ $\mathrm{CaO}$. The scattering lengths were taken to be equal to $b_{S i, O, A l, C a}=4.149,5.803,3.449$, $4.700 \mathrm{fm}$. 


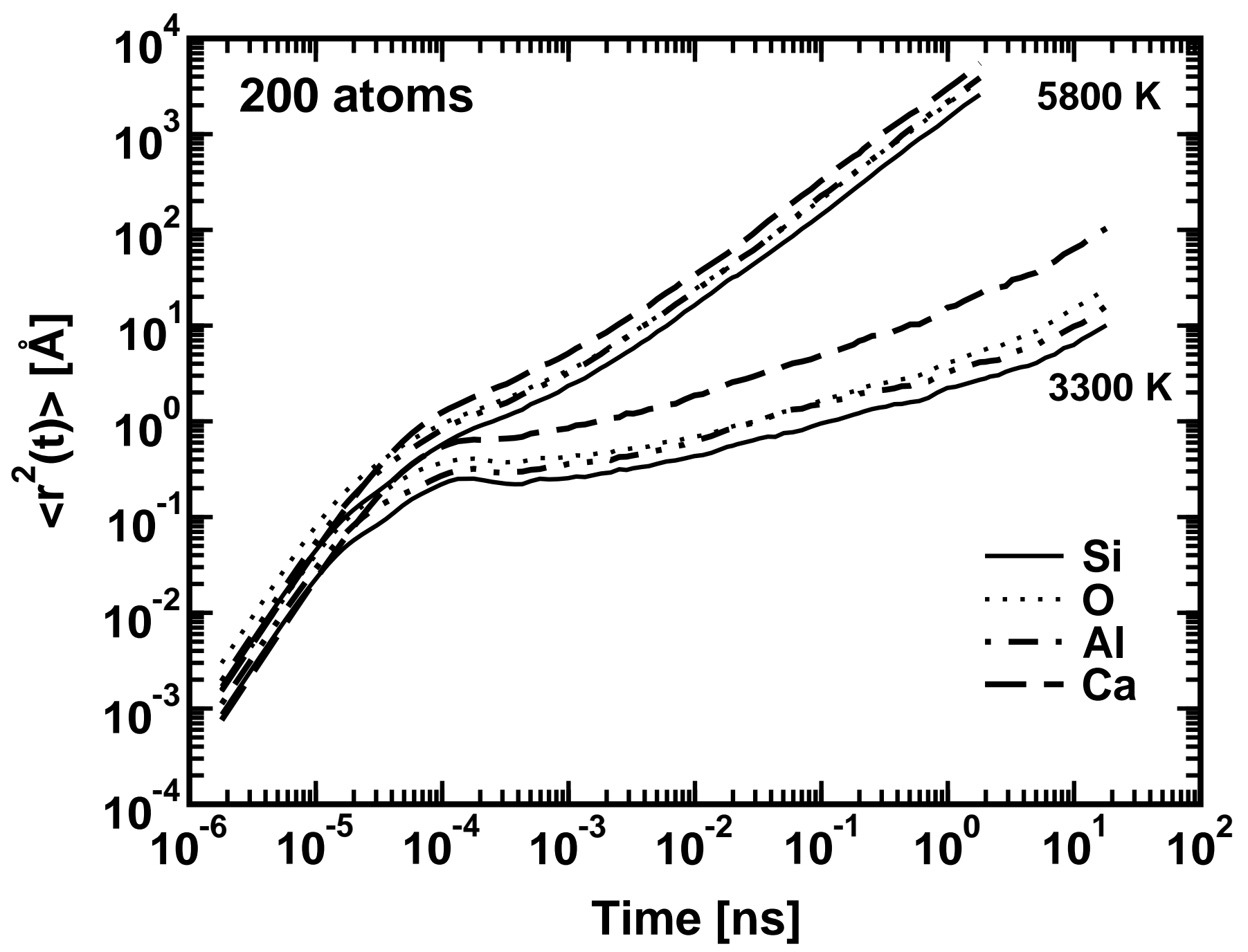

FIG. 1: 


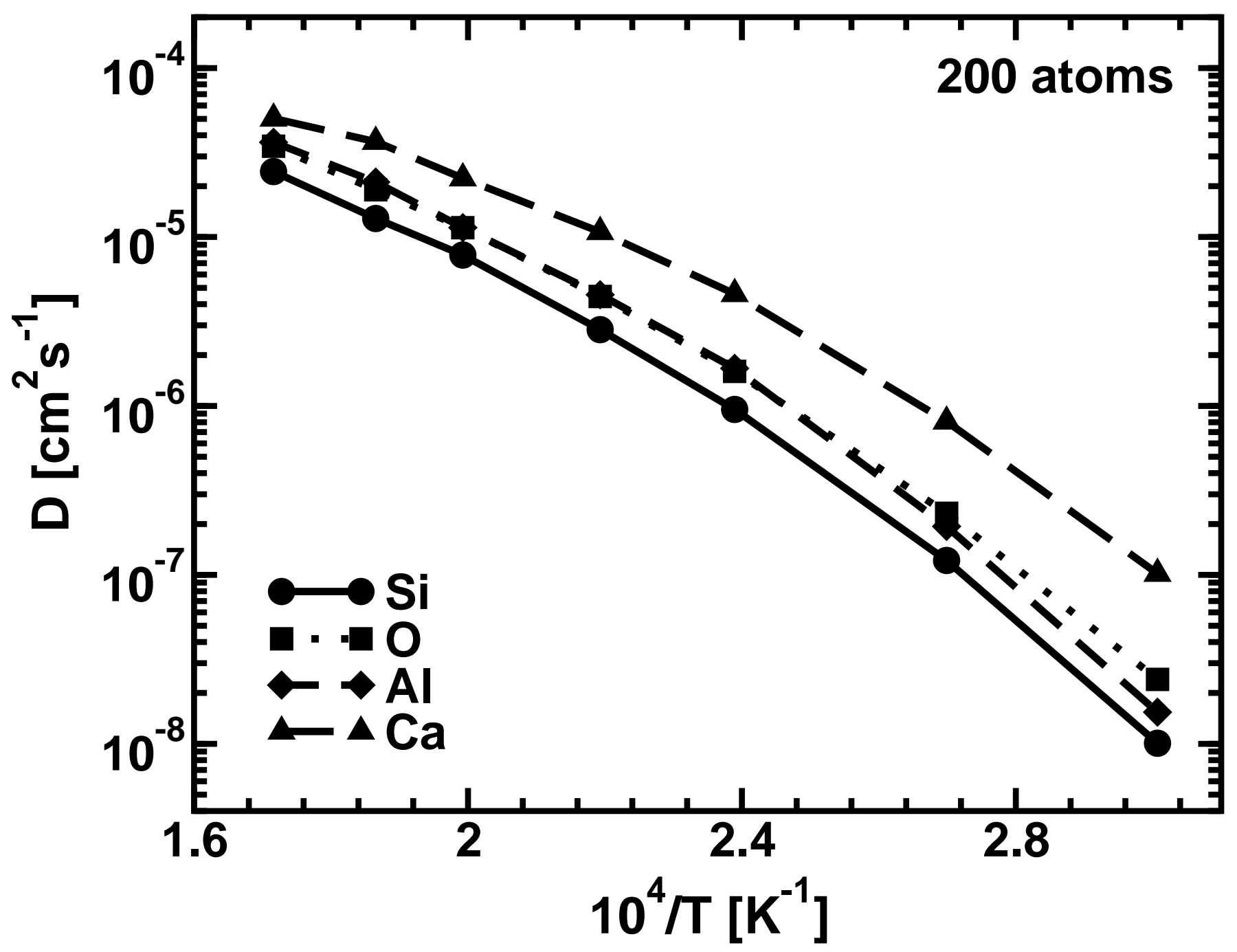

FIG. 2: 

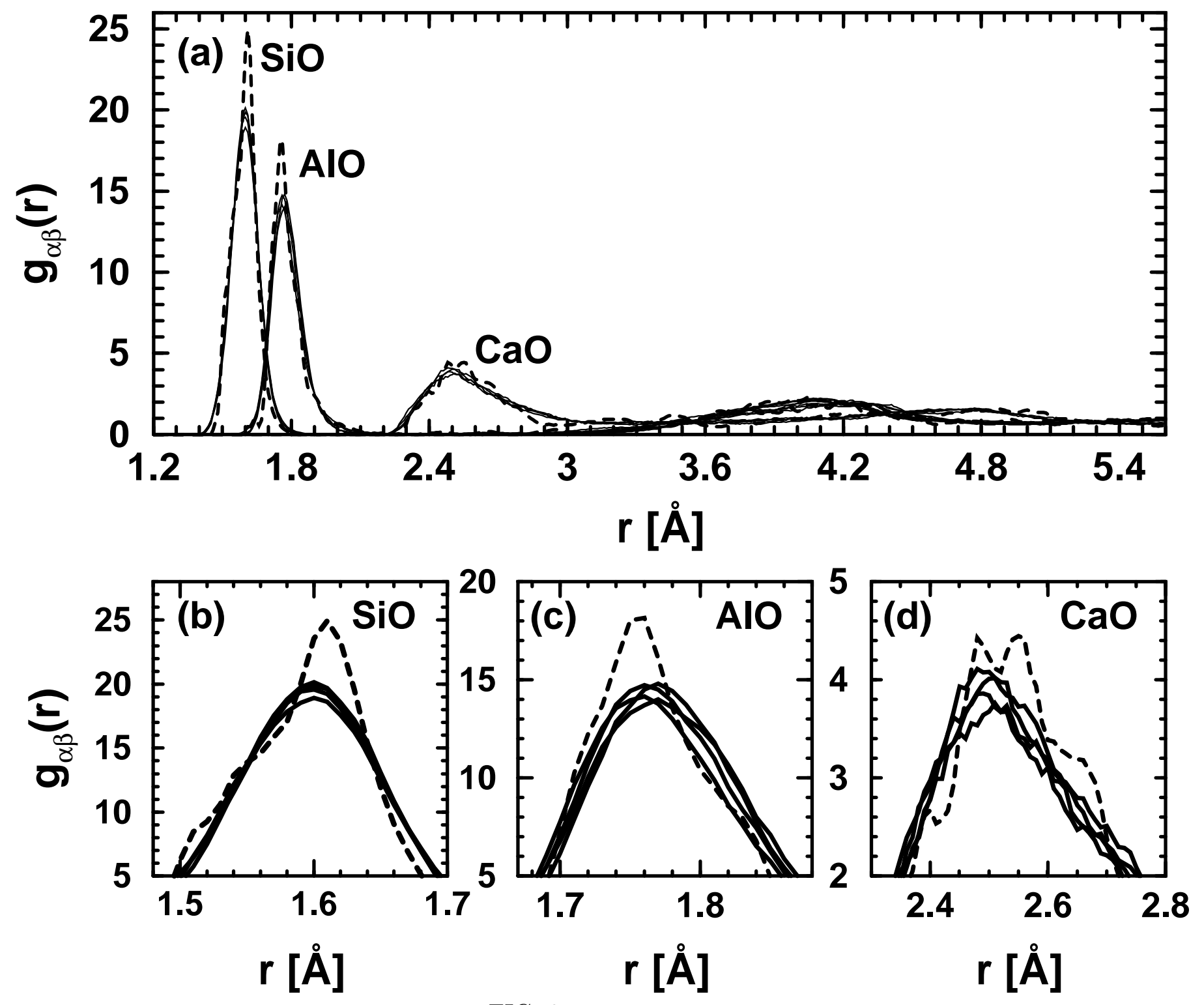

FIG. 3: 

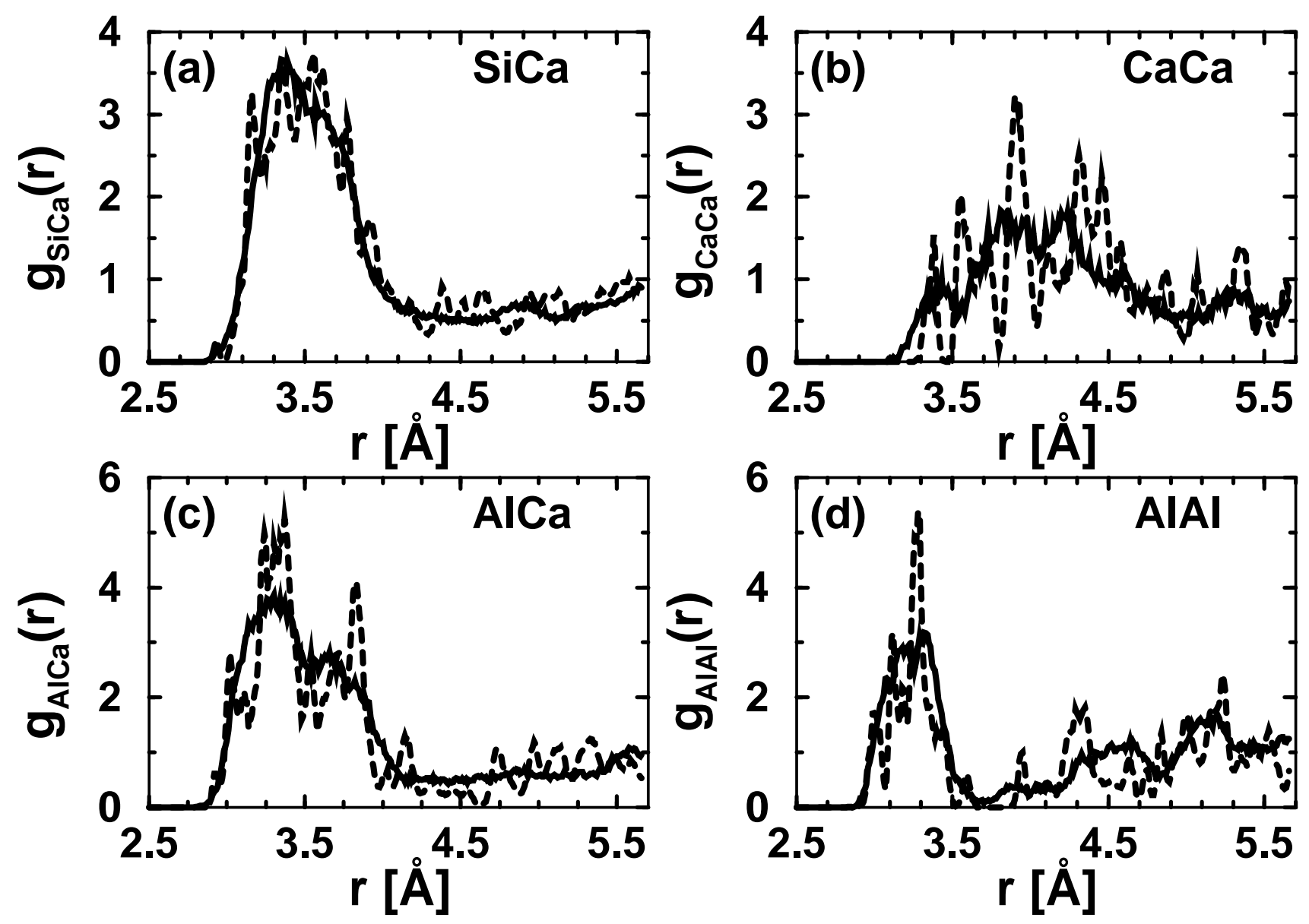

FIG. 4: 


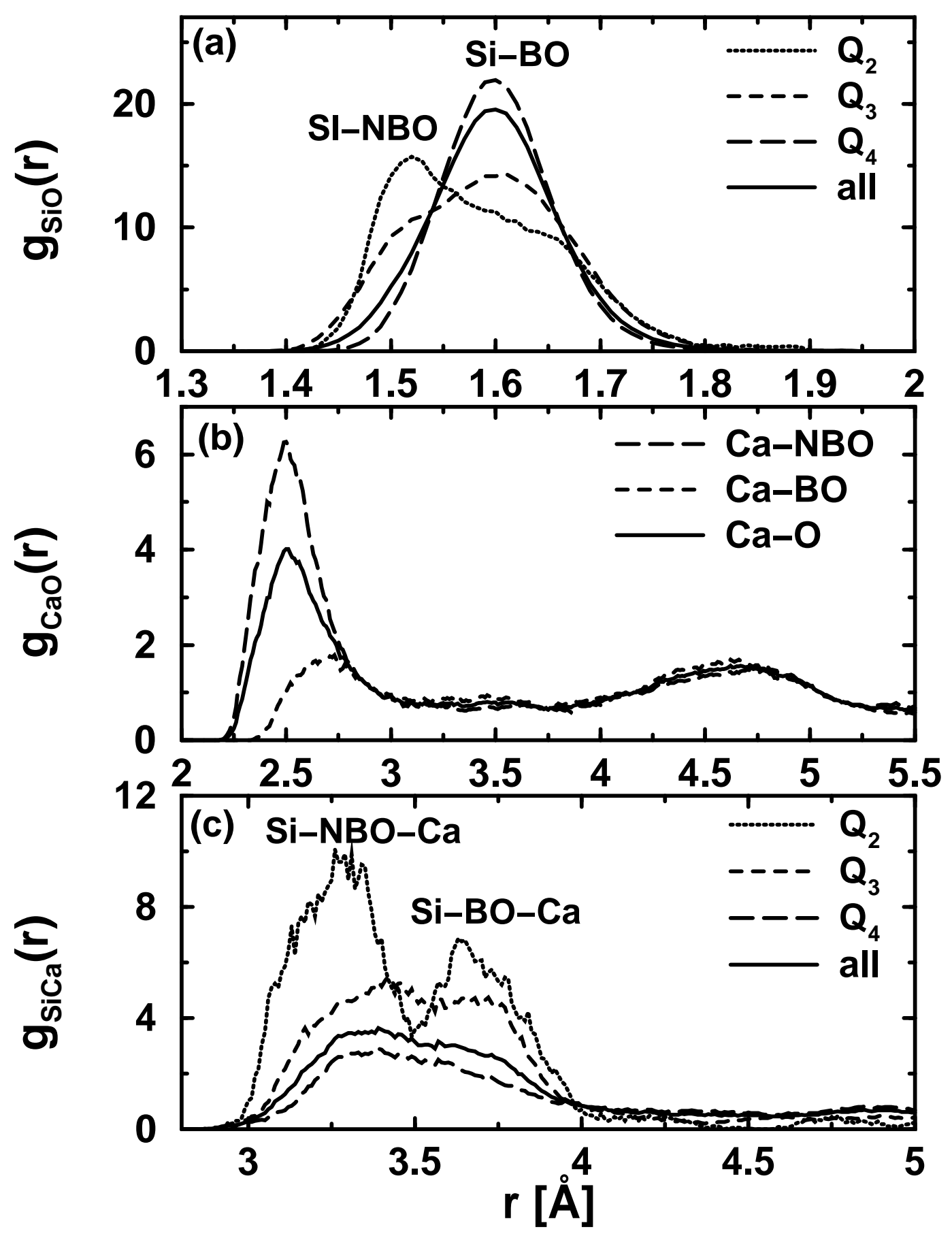

FIG. 5: 


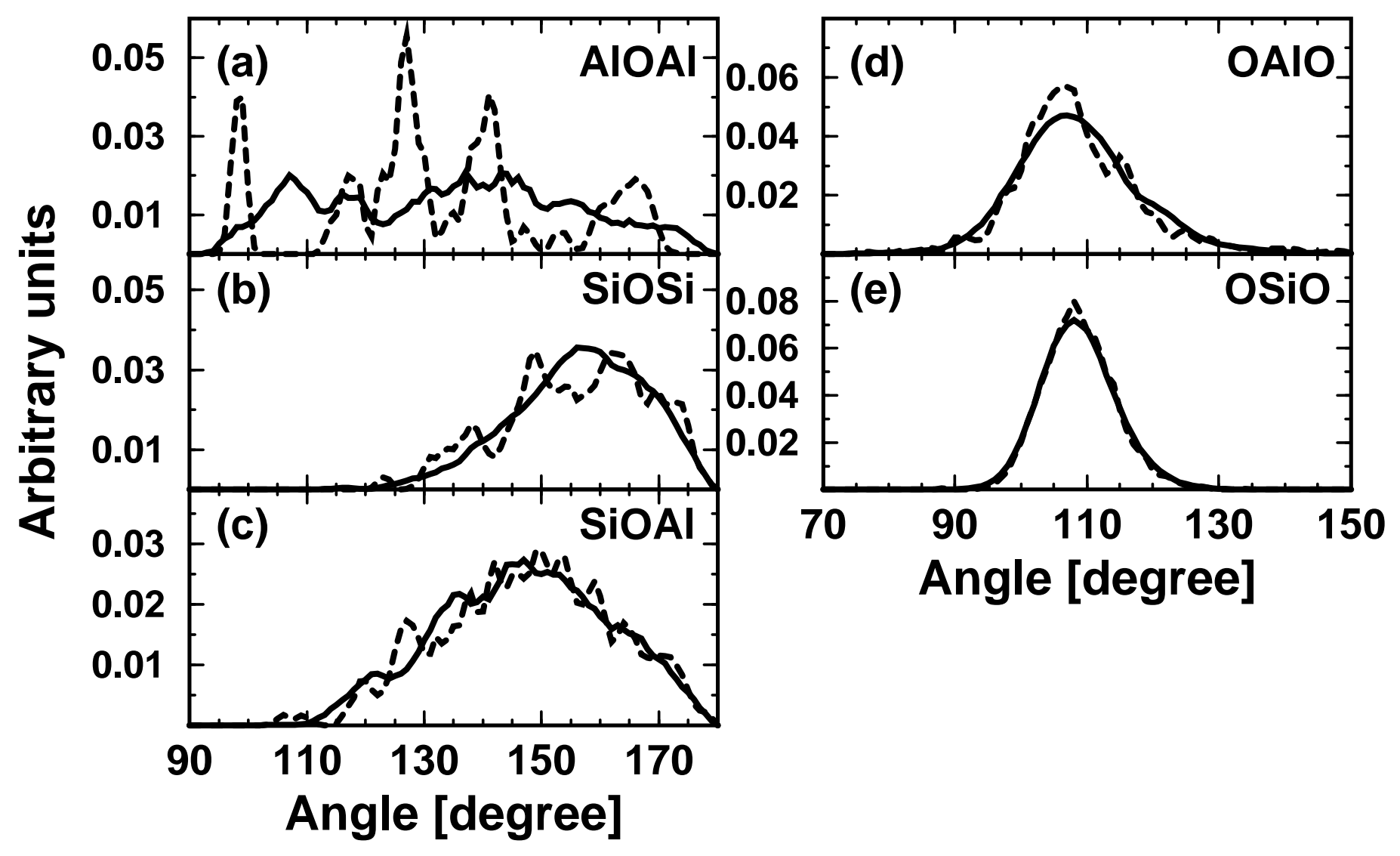

FIG. 6: 

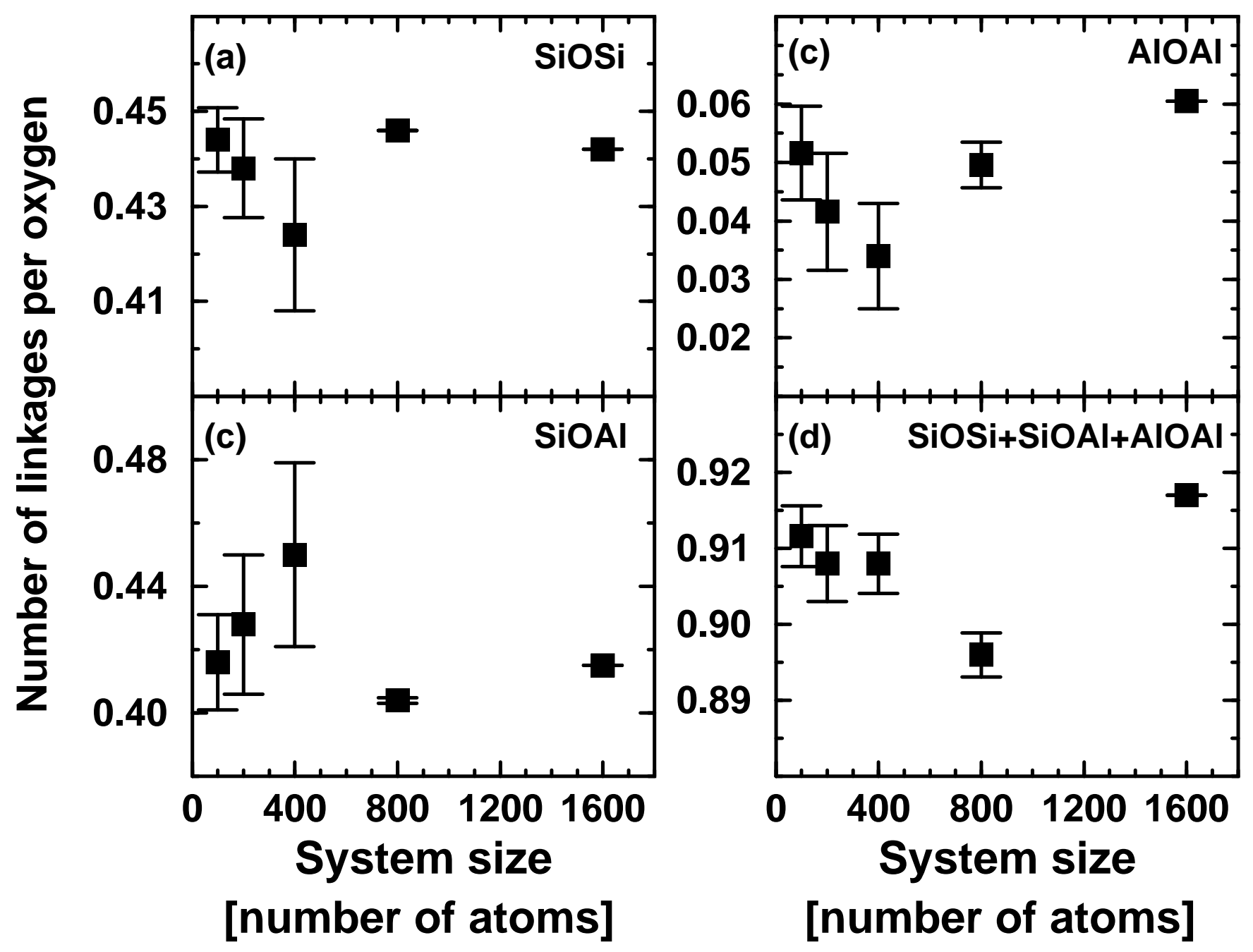

FIG. 7: 


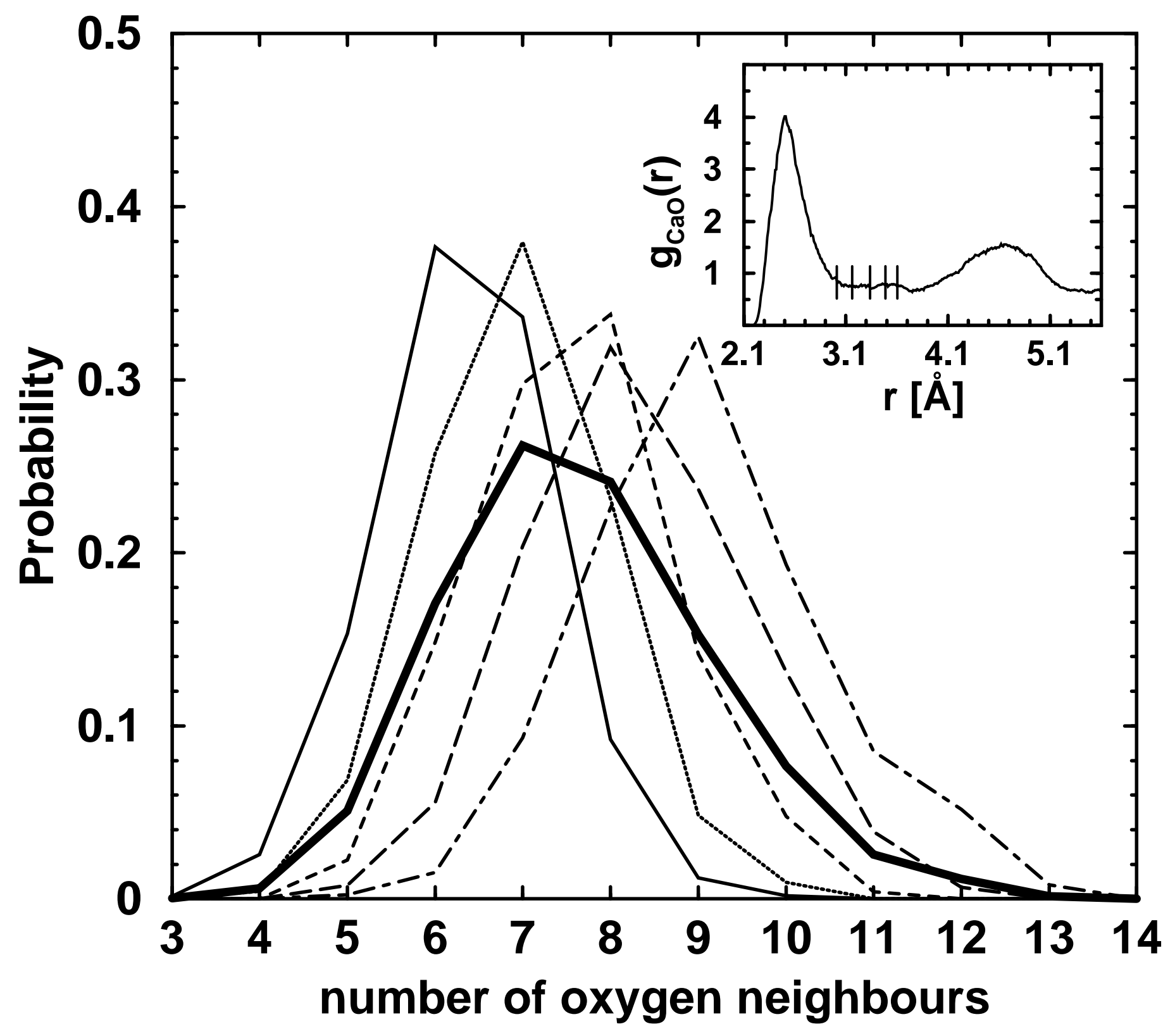

FIG. 8: 


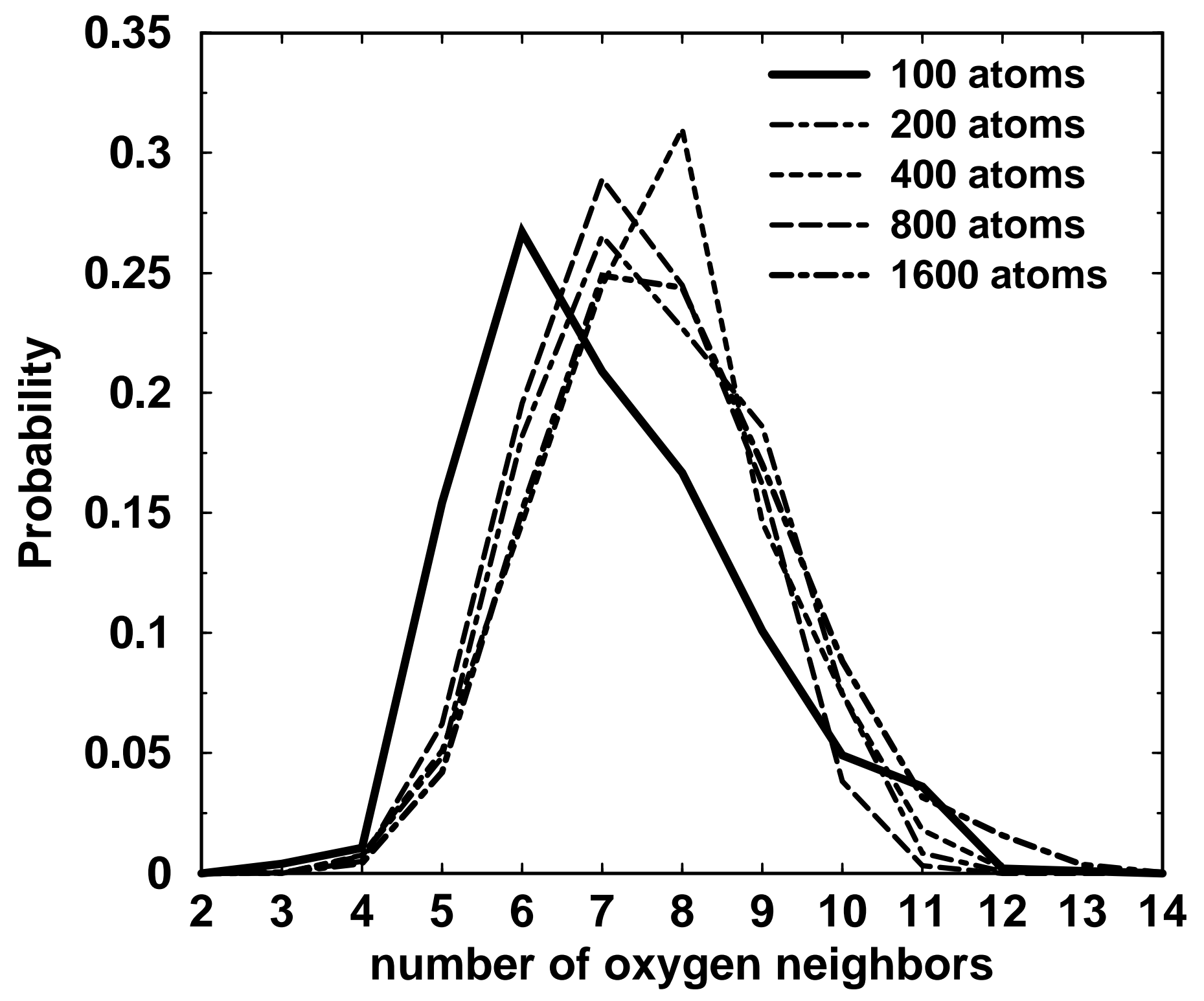

FIG. 9: 


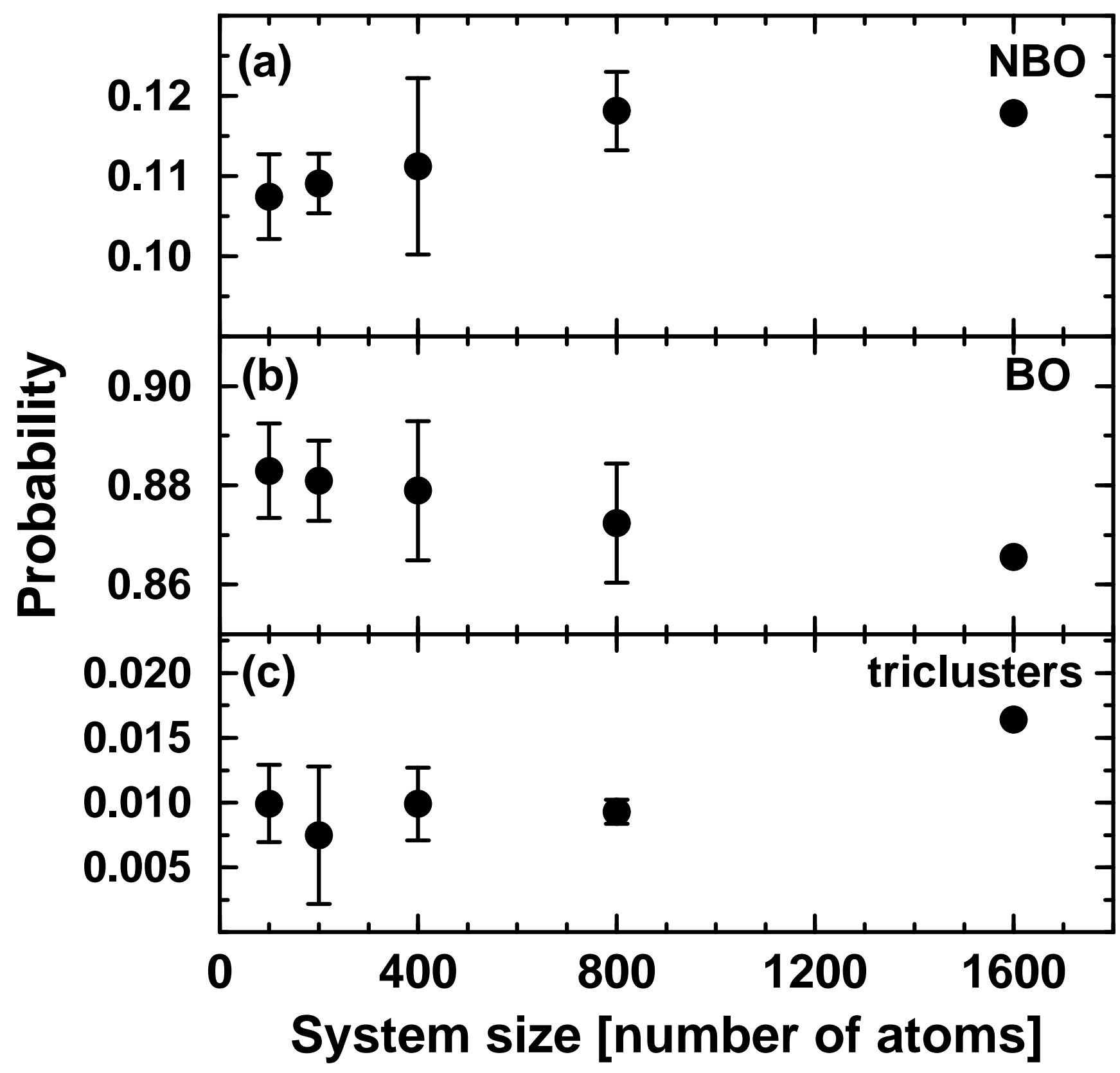

FIG. 10: 


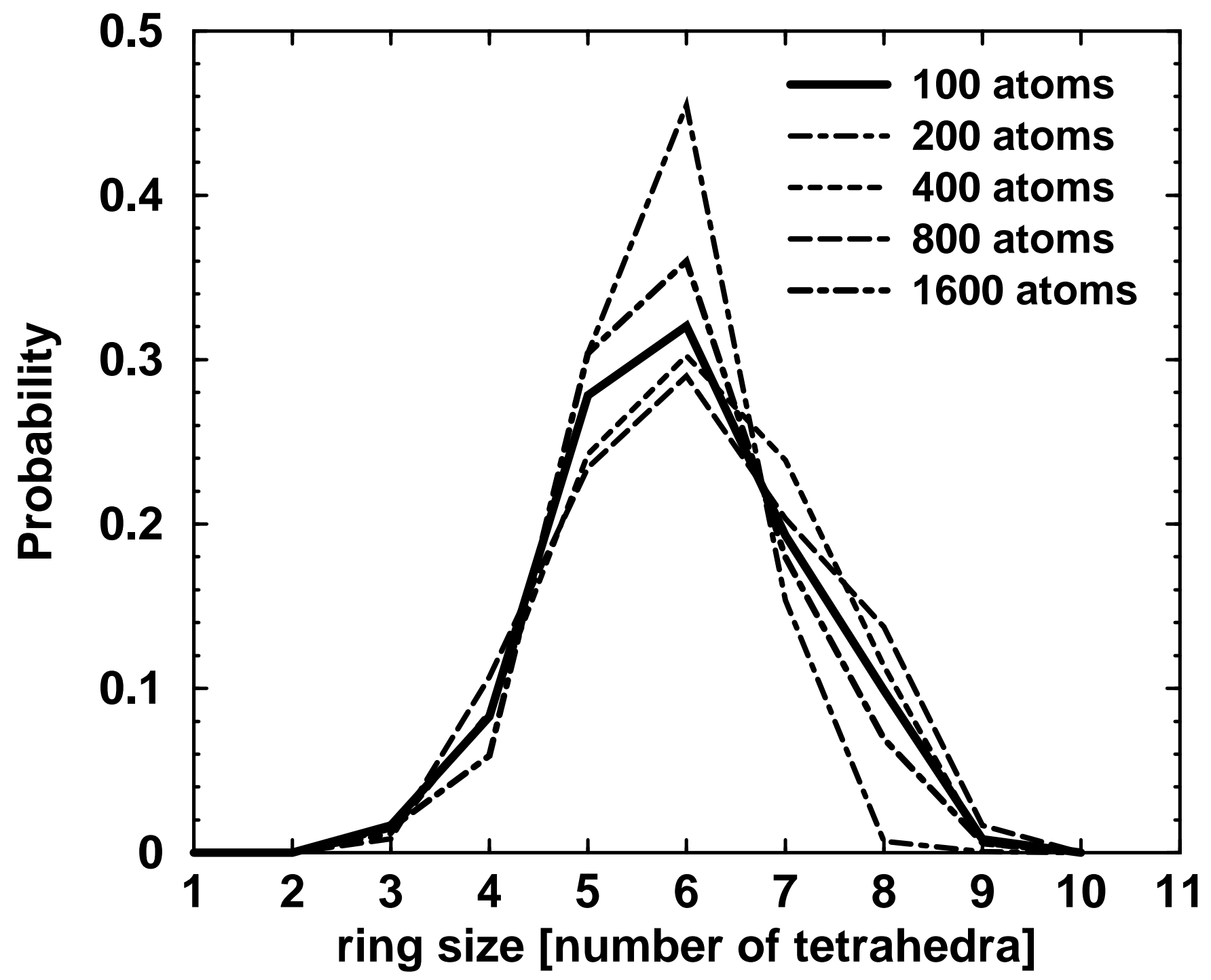

FIG. 11: 


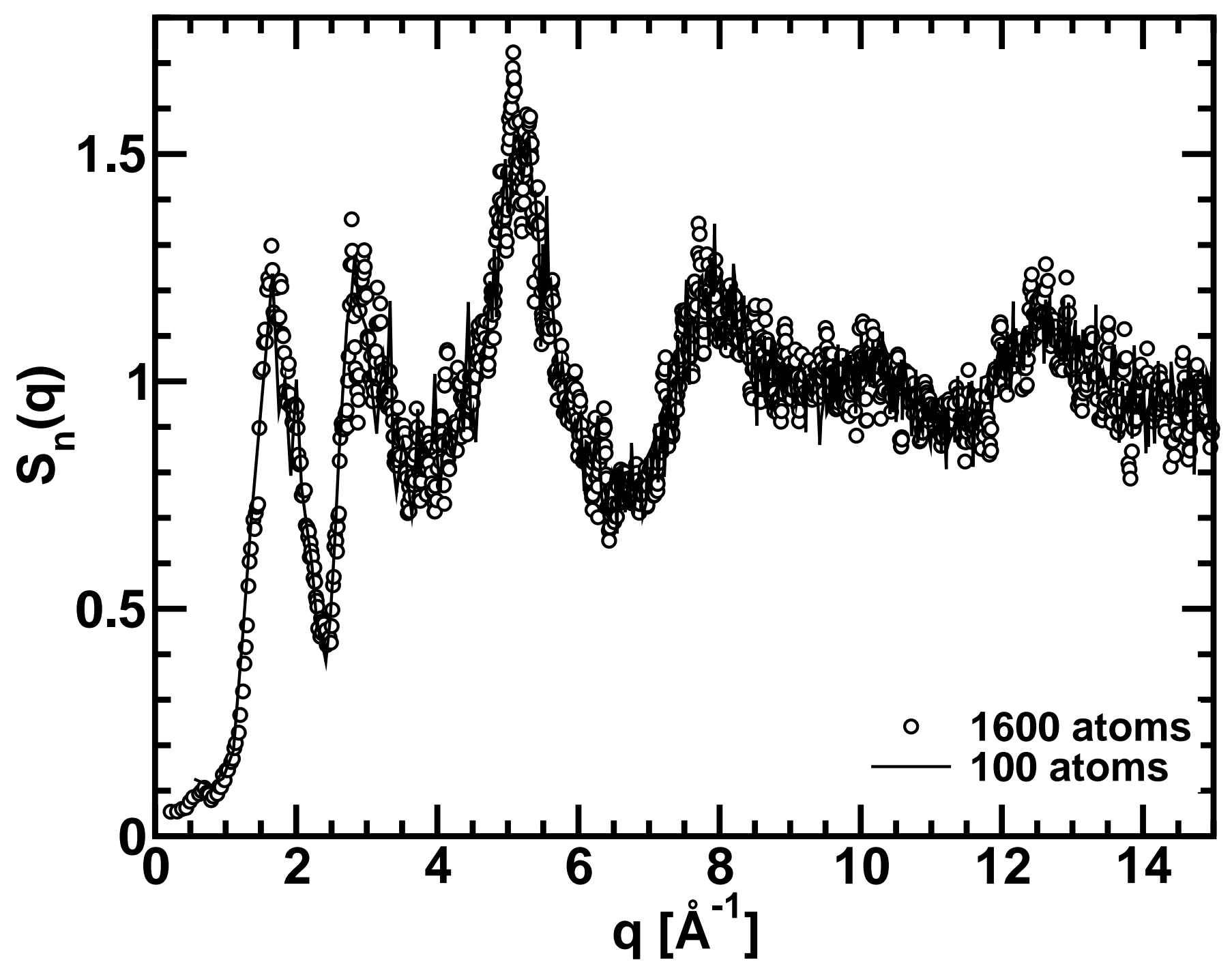

FIG. 12: 


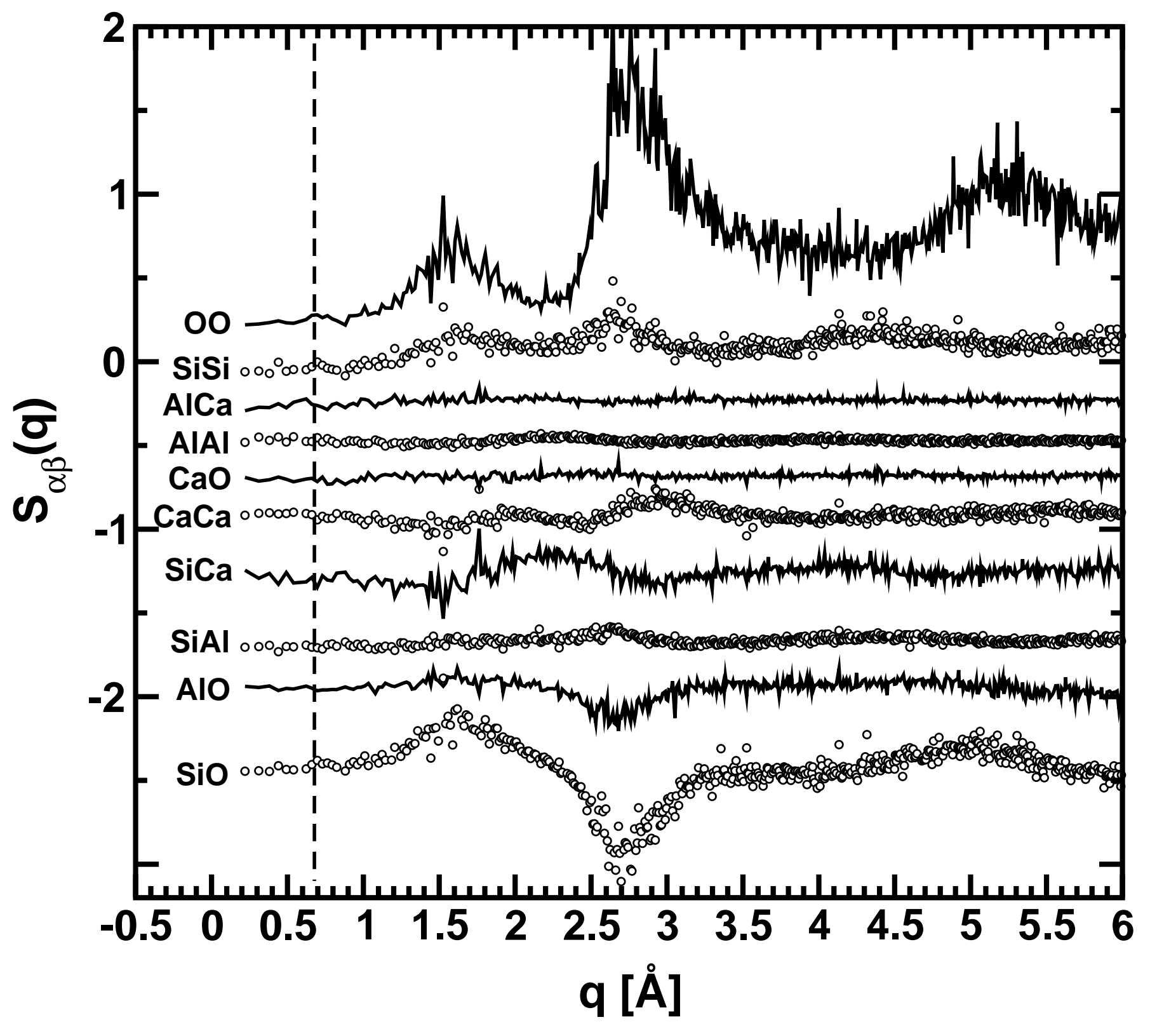

FIG. 13: 


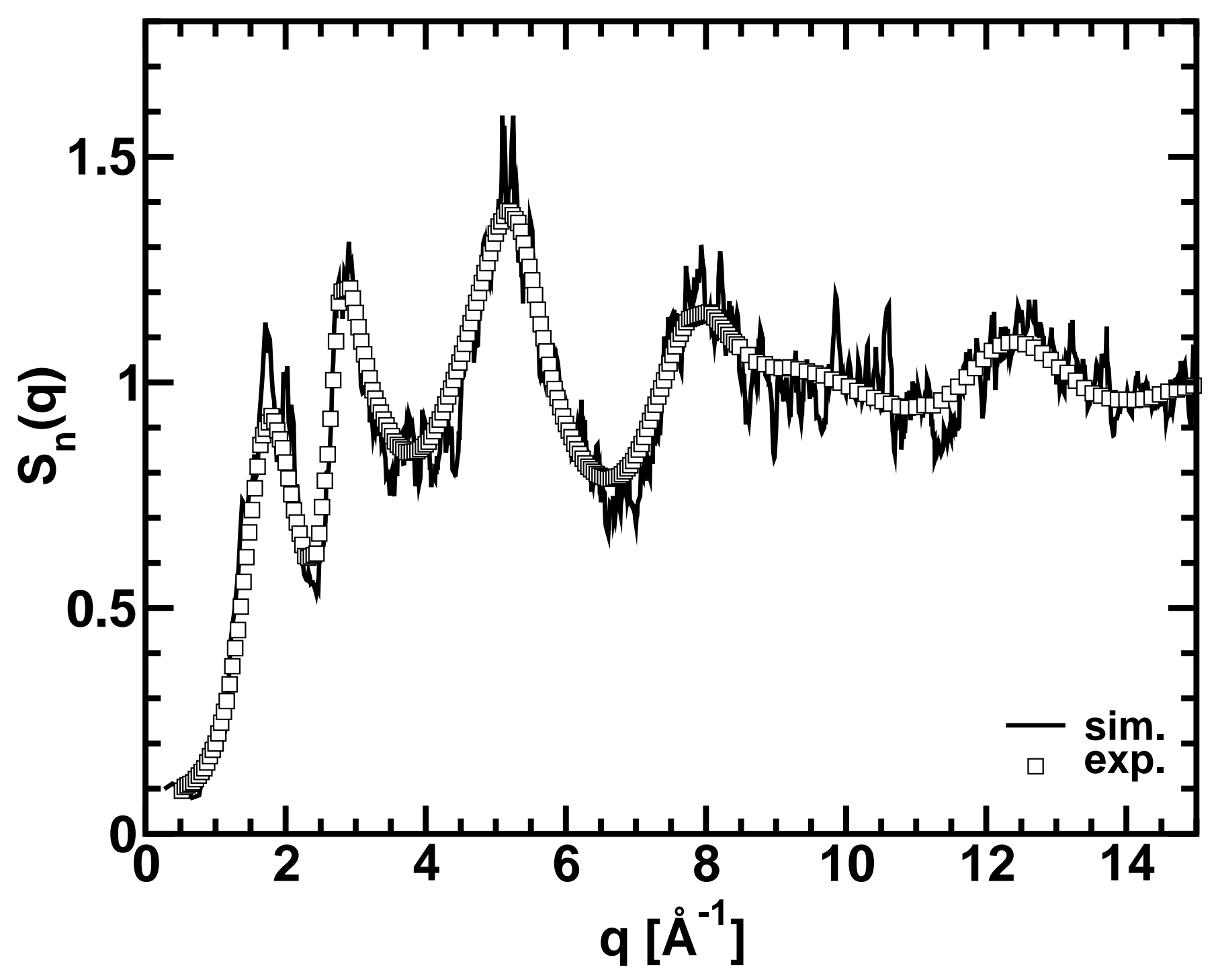

FIG. 14: 